\title{
AN INTEGRATED PICTURE FUZZY ANP-TODIM MULTI-CRITERIA DECISION-MAKING APPROACH FOR TOURISM ATTRACTION RECOMMENDATION
}

\author{
Chao TIAN 2 , Juan-juan PENG ${ }^{1 *}$ \\ ${ }^{1}$ School of Information, Zhejiang University of Finance and Economics, Hangzhou 310018, China \\ ${ }^{2}$ School of Accounting, Zhejiang University of Finance and Economics, Hangzhou 310018, China
}

Received 15 November 2018; accepted 22 September 2019

\begin{abstract}
In this paper, the picture fuzzy score and accuracy function are first defined. Then, a corresponding comparative method between two picture fuzzy numbers (PFNs) is developed. Next, a novel normalized picture fuzzy distance measure between two PFNs is disclosed, and part of the characteristics of the proposed distance measure are discussed. Afterwards, on the basis of the analytic network process (ANP) and an Acronym in Portuguese of Interactive and Multi-Criteria Decision-Making (TODIM) methods, an integrated ANP-TODIM approach is developed to resolve multi-criteria decision-making (MCDM) where the weights of the criteria are fully unknown. We use ANP approach to decide the weights of criteria on the basis of expert mean assessment method, and TODIM is utilized to obtain the ranking of alternatives. Finally, an illustrative example of an optimal tourism attraction recommendation is provided to testify applicability of the developed decision-making method and prove that its results are effective and reasonable.
\end{abstract}

Keywords: multi-criteria decision-making, picture fuzzy numbers, ANP-TODIM, picture fuzzy distance.

JEL Classification: D81, C61, Q65.

\section{Introduction}

In real decision-making process, to provide an accurate assessment regarding imprecise, incomplete or uncertain information by using a single crisp value is challenging for decisionmakers (DMs). Fuzzy sets (FSs) (Zadeh, 1965), where the membership degree is a real number in $[0,1]$, are important for handling multi-criteria decision-making (MCDM) problems. But FSs are not able to fully solve uncertainty in various real-life decision-making problems. When a DM aims to make an evaluation on an explanation, he/she may state that the possibility of the explanation being true is 0.5 and false is 0.3 . Hence, FSs cannot truly describe inconsistent information in such problems. In order to overcome this shortcoming, intu-

${ }^{\star}$ Corresponding author. E-mail: pengjj81@csu.edu.cn

This is an Open Access article distributed under the terms of the Creative Commons Attribution License (http://creativecommons. org/licenses/by/4.0/), which permits unrestricted use, distribution, and reproduction in any medium, provided the original author and source are credited. 
itionistic fuzzy sets (IFSs) (Atanassov, 1986) and their extensions, which can be represented as membership and non-membership, have recently attracted scholars' attention, and have been extensively applied to MCDM problems as well (Liu \& Wang, 2007; Xu, 2007; Wang \& Liu, 2012; Wang, Han, \& Zhang, 2014; Yu, J. Wang, \& J. Q. Wang, 2018; Hu, Zhang, Yang, Liu, \& Chen, 2018; Yang, Hu, Liu, \& Chen, 2018; Debnath, Bandyapadhyay, Roy, \& Kar, 2018; Chatterjee \& Kar, 2018a, 2018b). In particular, entropy, cross-entropy and similarity measures for IFSs have been utilized to deal with MCDM problems in an uncertain environment (Li \& Cheng, 2002; Yang, Gong, Wang, \& An, 2014; Mishra, Jain, \& Hooda, 2017; Joshi, Kumar, Gupta, \& Kaur, 2017; Ngan, Son, Cuong, \& Ali, 2018; Shen, Li, \& Wang, 2019).

Picture fuzzy sets (PFSs) were initially presented by Cuong and Kreinovich (2013) on the basis of IFSs, and are expressed by degrees of membership, neutral membership, and nonmembership. Additionally, PFSs can precisely describe a DMs' opinions, including yes, abstain, no, and refusal, that can avoid any missing information required for evaluation purposes and make data to be more reliable and adaptable with an actual decision-making environment than IFSs. Recently, PFSs-based studies have focused on their extensions and decisionmaking methods to deal with various MCDM and clustering analysis problems (Singh, 2015; Wei, 2016; Thong, 2016a, 2016b; Son, 2017; Zhang, Wang, \& Hu, 2018; L. Wang, Peng, \& J. Q. Wang, 2018; L. Wang, Zhang, J. Q. Wang, \& Wu, 2019; L. Wang, X. K. Wang, Peng, \& J. Q. Wang, 2020). For example, Wei (2016) defined a cross-entropy measure of PFSs and accordingly applied it to the selection of enterprise resource planning systems. Furthermore, Singh (2015) developed the coefficient and Thong (2016a, 2016b) presented a novel clustering analysis algorithm using picture fuzzy information; Son (2017) proposed the distance and similarity measures of PFSs and used them to clustering analysis; Wang et al. (2018) investigated the picture fuzzy Bonferroni mean distance, whilst V. D. Nguyen and X. T. Nguyen (2018) developed some distances of PFSs, and then applied them to solve MCDM problems; Tian, Peng, S. Zhang, W. Y. Zhang, and Wang (2019) and R. Wang, J. Wang, Gao, and Wei (2019) defined picture fuzzy geometric aggregation operators and picture fuzzy Muirhead mean operators, respectively.

The analytic network process (ANP) is an extension of analytic hierarchy process (AHP) (Saaty, 1996). Several studies have applied fuzzy ANP and other fuzzy MCDM methods to handle various situations and problems. For instance, Chang, S. K. Liao, Tseng, and C. Y. Liao (2015) developed an ANP-TOPSIS (Technique for Order Preference by Similarity to Ideal Solution) method for a location selection, and $\mathrm{Wu}$, Zhang, $\mathrm{Xu}$, and $\mathrm{Li}$ (2018) proposed a fuzzy ANP-VIKOR (VIseKriterijumska Optimizacija I KOmpromisno Resenje) approach for attraction selection, and Debnath, Roy, and Kar (2018) developed an integrated method to measure corporate social responsibility based on ANP and a process-based balance scorecard model.

Furthermore, Gomes and Lima (1992a, 1992b) used prospect theory (Kahneman \& Tversky, 1979) to propose the TODIM (an Acronym in Portuguese of Interactive and Multi-Criteria Decision-Making) method, taking a DMs' bounded rationality into account. Subsequently, Zhang and $\mathrm{Xu}$ (2014) and Peng, Wang, and Wu (2016) developed an extended hesitant fuzzy TODIM method. Moreover, it has been extended to other types of information, including neutrosophic sets (Pramanik, Dalapati, Alam, \& Roy, 2018), multi-valued neutrosophic sets 
(Ji, Zhang, \& Wang, 2018), intuitionistic linguistic sets (Yu et al., 2018), and probabilistic interval-valued hesitant fuzzy sets (Zhang, J. Q. Wang, \& T. L. Wang, 2019).

However, the above-mentioned methods contain a number of deficiencies or limitations. Firstly, the existing picture fuzzy distance measures cannot consider cross-evaluation among positive degree, neutral degree, and negative degree, which may restrict their practical application. Secondly, the aforementioned MCDM methods with picture fuzzy information are fully on the basis of rationality, while a DM is often influenced by several factors, such as psychology, personality, environment, and risk preference. Thirdly, the majority of existing picture MCDM methods cannot consider interdependence between criteria, as well as being unable to manage particular situations, in which the weight information is fully unknown. Thus, this study mainly aims to (1) Define the score and accuracy functions of PFNs, and develop the corresponding comparison method; (2) Propose a novel picture fuzzy distance measure, that can avoid the first shortcoming, i.e., a normalized picture fuzzy distance to take cross-evaluation into account among three membership degrees, and make the distance to be more reasonable and flexible; (3) Investigate an integrated ANP-TODIM approach to avoid the second and third shortcomings, which can consider a DMs' bounded rationality. Importantly, the proposed method is highly appropriate to solve MCDM problems with interdependence between criteria and fully unknown weight information.

The study is arranged as follows. Section 1 reviews some definitions and the comparative method is defined according to the score and accuracy functions. In Section 2, a novel normalized picture fuzzy distance measure is presented and the corresponding properties are discussed. Section 3 develops an integrated ANP-TODIM MCDM approach with picture fuzzy information. A theoretical case is shown in Section 4 to testify the feasibility and validity of the new method. Eventually, last section draws the conclusions.

\section{PFSs}

Some definitions related to PFSs are reviewed. Furthermore, the score and accuracy functions and a comparative method for two PFNs are presented.

Definition 1 (Cuong \& Kreinovich, 2013). Let $X$ be the universe, and a PFS $\widehat{\Gamma}$ can be given by:

$$
\widehat{\Gamma}=\left\{\left(x, \overline{\overline{P O}}_{\Gamma}(x), \overline{\overline{N U}}_{\bar{\Gamma}}(x), \overline{\overline{N G}}_{\Gamma}(x)\right) \mid x \in X\right\} .
$$

Where $\overline{\overline{P O}} \bar{\Gamma}(x) \in[0,1]$ denote the positive membership degree of $\widehat{\Gamma}, \overline{\bar{N}}{ }_{\Gamma}(x) \in[0,1]$ stands the neutral membership degree of $\widehat{\Gamma}$, and $\overline{\overline{N G}} \bar{\Gamma}(x) \in[0,1]$ represents the negative membership degree of $\hat{\Gamma}$; moreover, $\overline{\overline{P O}} \bar{\Gamma}(x), \overline{\overline{N U}} \bar{\Gamma}(x)$ and $\overline{\overline{N G}} \bar{\Gamma}(x)$ meet the condition that $0 \leq \overline{\overline{P O}}_{\Gamma}(x)+\overline{\overline{N U}}_{\Gamma}(x)+\overline{\overline{N G}}_{\Gamma}(x) \leq 1$ for any $x \in X$. Then, $\overline{\overline{R F}}_{\Gamma}(x)=1-\left(\overline{\overline{P O}}_{\bar{\Gamma}}(x)+\overline{\overline{N U}}_{\bar{\Gamma}}(x)+\right.$ $\overline{\overline{N G}}(x))$ denotes the refusal membership degree of the element $x$ to subset $\widehat{\Gamma}$. For convenience, $\widehat{\Gamma}=(\overline{\overline{P O}} \bar{\Gamma}, \overline{\overline{N U}} \bar{\Gamma}, \overline{\overline{N G}} \widehat{\Gamma})$ denotes picture fuzzy number $(\mathrm{PFN})$.

Definition 2 (Cuong \& Kreinovich, 2013). Let $\widehat{\Gamma}, \widehat{\Gamma}_{1}$ and $\widehat{\Gamma}_{2}$ be three PFNs, and the corresponding operations are given by: 


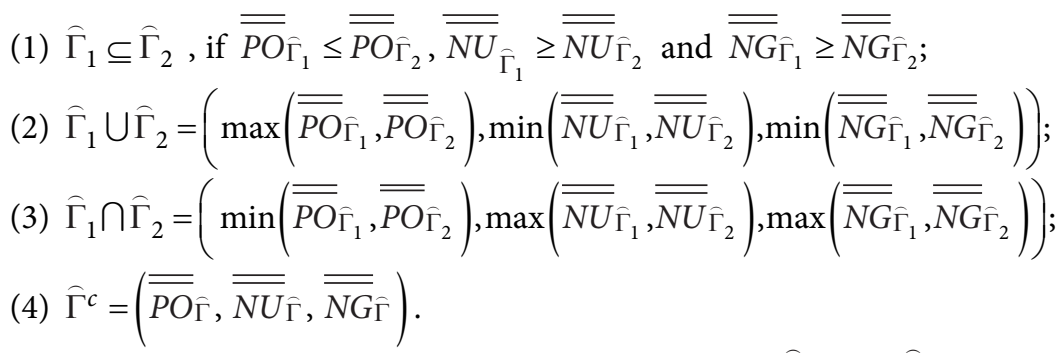

Definition 3 (V. D. Nguyen, \& X. T. Nguyen, 2018). Let $\widehat{\Gamma}_{1}$ and $\widehat{\Gamma}_{2}$ be two PFNs. Then, some picture fuzzy distance measures of $\widehat{\Gamma}_{1}$ and $\widehat{\Gamma}_{2}$ can be defined in the following:

(1) $D_{H}\left(\widehat{\Gamma}_{1}, \widehat{\Gamma}_{2}\right)=\frac{1}{3}\left(\left|\overline{\overline{P O}} \widehat{\Gamma}_{1}-\overline{\overline{P O}}_{\Gamma_{2}}\right|+\left|\overline{\overline{N U}} \widehat{\Gamma}_{1}-\overline{\overline{N U}}_{\Gamma_{2}}\right|+\left|\overline{\overline{N G}} \widehat{\Gamma}_{1}-\overline{\overline{N G}} \bar{\Gamma}_{2}\right|\right)$;

(2) $D_{E}\left(\widehat{\Gamma}_{1}, \widehat{\Gamma}_{2}\right)=\left(\left|\overline{\overline{P O}} \widehat{\Gamma}_{1}-\overline{\overline{P O}} \widehat{\Gamma}_{2}\right|^{2}+\left|\overline{\overline{N U}} \bar{\Gamma}_{1}-\overline{\overline{N U}} \widehat{\Gamma}_{2}\right|^{2}+\left|\overline{\overline{N G}} \widehat{\Gamma}_{1}-\overline{\overline{N G}} \widehat{\Gamma}_{2}\right|^{2}\right)^{1 / 2}$;

(3) $D_{H}^{m}\left(\widehat{\Gamma}_{1}, \widehat{\Gamma}_{2}\right)=\max \left(\left|\overline{\overline{P O}} \bar{\Gamma}_{1}-\overline{\overline{P O}} \widehat{\Gamma}_{2}\right|,\left|\overline{\overline{N U}} \bar{\Gamma}_{1}-\overline{\overline{N U}} \bar{\Gamma}_{2}\right|,\left|\overline{\overline{N G}} \widehat{\Gamma}_{1}-\overline{\overline{N G}} \widehat{\Gamma}_{2}\right|\right)$;

(4) $D_{E}^{m}\left(\widehat{\Gamma}_{1}, \widehat{\Gamma}_{2}\right)=\max \left(\left|\overline{\overline{P O}} \widehat{\Gamma}_{1}-\overline{\overline{P O}}_{\Gamma_{2}}\right|^{2},\left|\overline{\overline{N U}} \bar{\Gamma}_{1}-\overline{\overline{N U}} \widehat{\Gamma}_{2}\right|^{2},\left|\overline{\overline{N G}} \bar{\Gamma}_{1}-\overline{\overline{N G}} \widehat{\Gamma}_{2}\right|^{2}\right)^{1 / 2}$.

However, the picture fuzzy distances presented in Definition 3 are not fully consistent with real conditions in some particular cases. In the following, an example is shown to demonstrate the restriction of those distances.

Example 1. Let $\widehat{\Gamma}_{1}=(0.5,0,0.5), \widehat{\Gamma}_{2}=(0.4,0,0.6)$, and $\widehat{\Gamma}_{3}=(0.4,0,0.4)$ be three PFNs. According to the ten-people-voting model developed by Wang and Xin (2005), $\widehat{\Gamma}_{1}$ denotes five people voting "Yes", five people voting "No", and there is no people who abstain or refuse; $\widehat{\Gamma}_{2}$ represents four people voting "Yes", six people voting "No", and there is no people who abstain or refuse, while $\widehat{\Gamma}_{3}$ denotes four people voting "Yes", four people voting "No", two people voting "Refuse", and there is no people who abstain. Apparently, the difference between $\widehat{\Gamma}_{1}$ and $\widehat{\Gamma}_{2}$ is greater than that between $\widehat{\Gamma}_{1}$ and $\widehat{\Gamma}_{3}$ in real situations. However, based on the distances defined in Definition 3, we can obtain

$$
\begin{aligned}
& D_{H}\left(\widehat{\Gamma}_{1}, \widehat{\Gamma}_{2}\right)=\frac{1}{3}(|0.5-0.4|+|0-0|+|0.5-0.6|)=\frac{0.2}{3}=D_{H}\left(\widehat{\Gamma}_{1}, \widehat{\Gamma}_{3}\right), \\
& D_{E}\left(\widehat{\Gamma}_{1}, \widehat{\Gamma}_{2}\right)=D_{E}\left(\widehat{\Gamma}_{1}, \widehat{\Gamma}_{3}\right)=\sqrt{0.02}, D_{H}^{m}\left(\widehat{\Gamma}_{1}, \widehat{\Gamma}_{2}\right)=D_{H}^{m}\left(\widehat{\Gamma}_{1}, \widehat{\Gamma}_{3}\right)=0.1,
\end{aligned}
$$

and $D_{E}^{m}\left(\widehat{\Gamma}_{1}, \widehat{\Gamma}_{2}\right)=D_{E}^{m}\left(\widehat{\Gamma}_{1}, \widehat{\Gamma}_{3}\right)=0.1$.

The results indicate that the difference between $\widehat{\Gamma}_{1}$ and $\widehat{\Gamma}_{2}$ is as same as that between $\widehat{\Gamma}_{1}$ and $\widehat{\Gamma}_{3}$, contradicting real decision-making problems. Thus, the picture fuzzy distance measures presented in Definition 3 seem to be unreasonable in some particular cases.

Definition 4. Let $\widehat{\Gamma}=\left(\overline{\overline{P O}} \bar{\Gamma}, \overline{\overline{N U}_{\Gamma}}, \overline{\overline{N G}} \hat{\Gamma}\right)$ be a PFN, then we can define the score function as follows:

$$
S(\widehat{\Gamma})=\frac{1}{3}(\overline{\overline{P O}} \widehat{\Gamma}+1-\overline{\overline{N U}} \bar{\Gamma}+1-\overline{\overline{N G}} \widehat{\Gamma}),
$$


and the accuracy function is given by:

$$
A(\widehat{\Gamma})=\frac{1}{2}\left(\overline{\overline{N U}}_{\bar{\Gamma}}-\overline{\overline{N G}}_{\bar{\Gamma}}\right) .
$$

Definition 5. Let $\widehat{\Gamma}_{1}$ and $\widehat{\Gamma}_{2}$ be two PFNs, then we can define the comparative method for PFSs as follows:

(1) If $S\left(\widehat{\Gamma}_{1}\right)>S\left(\widehat{\Gamma}_{2}\right)$ or $S\left(\widehat{\Gamma}_{1}\right)=S\left(\widehat{\Gamma}_{2}\right)$ and $A\left(\widehat{\Gamma}_{1}\right)>A\left(\widehat{\Gamma}_{2}\right)$, then $\widehat{\Gamma}_{1} \succ \widehat{\Gamma}_{2}$;

(2) If $S\left(\widehat{\Gamma}_{1}\right)=S\left(\widehat{\Gamma}_{2}\right)$ and $A\left(\widehat{\Gamma}_{1}\right)=A\left(\widehat{\Gamma}_{2}\right)$, then $\widehat{\Gamma}_{1}=\widehat{\Gamma}_{2}$.

Example 2. Let $\widehat{\Gamma}_{1}=(0.6,0.2,0.1)$ and $\widehat{\Gamma}_{2}=(0.6,0.1,0.2)$ be two PFNs. Then, based on Definitions 4 and $5, S\left(\widehat{\Gamma}_{1}\right)=\frac{1}{3}(0.6+1-0.2+1-0.1)=0.767$ and $S\left(\widehat{\Gamma}_{2}\right)=\frac{1}{3}(0.6+1-0.1+$ $1-0.2)=0.767$ can be achieved. Since $A\left(\widehat{\Gamma}_{1}\right)=0.05>A\left(\widehat{\Gamma}_{2}\right)=-0.05$, thus we can obtain $\widehat{\Gamma}_{1} \succ \widehat{\Gamma}_{2}$. The results are consistent with our intuition as well.

\section{A novel normalized picture fuzzy distance measure}

Based on the intuitionistic fuzzy H-max distance defined by Ngan et al. (2018), a novel normalized picture fuzzy distance measure is herein proposed.

Definition 6. Let $\widehat{\Gamma}_{1}, \widehat{\Gamma}_{2}$, and $\widehat{\Gamma}_{3}$ be three PFNs. A mapping $\widehat{D}_{p f}: P F N \times P F N \rightarrow R$ is a normalized picture fuzzy distance measure when it meets the following axioms:

H1. $0 \leq \widehat{D}_{p f}\left(\widehat{\Gamma}_{1}, \widehat{\Gamma}_{2}\right) \leq 1$;

H2. $\widehat{D}_{p f}\left(\widehat{\Gamma}_{1}, \widehat{\Gamma}_{2}\right)=\widehat{D}_{p f}\left(\widehat{\Gamma}_{2}, \widehat{\Gamma}_{1}\right)$;

H3. $\widehat{D}_{p f}\left(\widehat{\Gamma}_{1}, \widehat{\Gamma}_{2}\right)=0$ if and only if $\widehat{\Gamma}_{1}=\widehat{\Gamma}_{2}$;

H4. If $\widehat{\Gamma}_{1} \subseteq \widehat{\Gamma}_{2} \subseteq \widehat{\Gamma}_{3}$, then $\widehat{D}_{p f}\left(\widehat{\Gamma}_{1}, \widehat{\Gamma}_{3}\right) \geq \widehat{D}_{p f}\left(\widehat{\Gamma}_{1}, \widehat{\Gamma}_{2}\right)$ and $\widehat{D}_{p f}\left(\widehat{\Gamma}_{1}, \widehat{\Gamma}_{3}\right) \geq \widehat{D}_{p f}\left(\widehat{\Gamma}_{2}, \widehat{\Gamma}_{3}\right)$.

Definition 7. Let $\widehat{\Gamma}_{1}$ and $\widehat{\Gamma}_{2}$ be two PFNs. A novel picture fuzzy distance measure of $\widehat{\Gamma}_{1}$ and $\widehat{\Gamma}_{2}$ can be defined as:

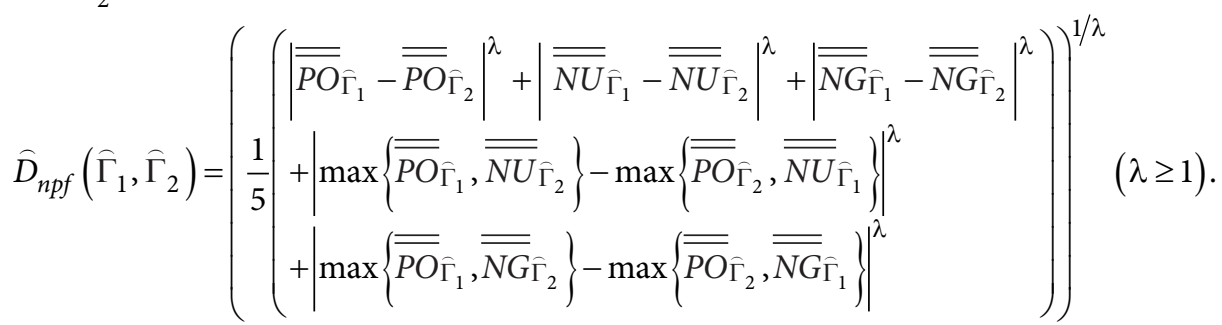

(1) In particular, if $\lambda=1$, then the novel picture fuzzy distance measure is reduced to a novel picture fuzzy Hausdorff distance measure, i.e.,

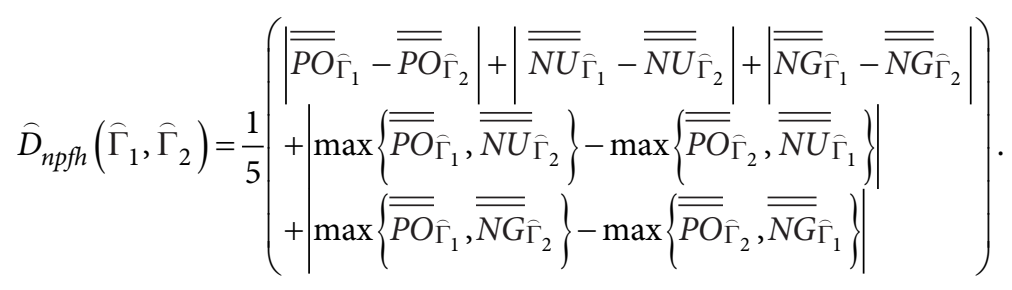


(2) If $\lambda=2$, then the novel picture fuzzy distance measure is reduced to a novel picture fuzzy Euclidean distance measure, i.e.,

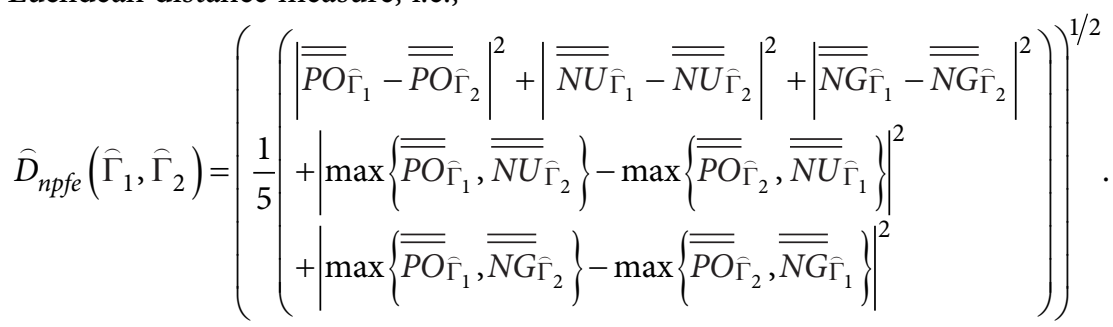

Example 3. Based on Example 1 and the novel picture fuzzy Hausdorff distance developed in Definition 7, we have

$$
\begin{aligned}
& \widehat{D}_{n p f h}\left(\widehat{\Gamma}_{1}, \widehat{\Gamma}_{2}\right)=\frac{1}{5}(|0.5-0.4|+|0-0|+|0.5-0.6|+|\max \{0.5,0.6\}-\max \{0.4,0.5\}|+ \\
& |\max \{0.5,0\}-\max \{0.4,0\}|)=0.08 .
\end{aligned}
$$

Similarly, $\widehat{D}_{n p f h}\left(\widehat{\Gamma}_{1}, \widehat{\Gamma}_{3}\right)=0.06$ can be obtained. As $\widehat{D}_{n p f h}\left(\widehat{\Gamma}_{1}, \widehat{\Gamma}_{2}\right)>D_{n p f h}\left(\widehat{\Gamma}_{1}, \widehat{\Gamma}_{3}\right)$, thus the difference between $\widehat{\Gamma}_{1}$ and $\widehat{\Gamma}_{2}$ is greater than that between $\widehat{\Gamma}_{1}$ and $\widehat{\Gamma}_{3}$. The result is consistent with the real decision-making problems. Consequently, the proposed distance is more effective than that presented in Definition 3.

Theorem 1. The novel picture fuzzy distance measure presented in Definition 7 is a normalized distance measure of PFNs, i.e., $\widehat{D}_{n p f}\left(\widehat{\Gamma}_{1}, \widehat{\Gamma}_{2}\right)$ satisfies the following axioms:

H1. $0 \leq \widehat{D}_{n p f}\left(\widehat{\Gamma}_{1}, \widehat{\Gamma}_{2}\right) \leq 1$;

H2. $\widehat{D}_{n p f}\left(\widehat{\Gamma}_{1}, \widehat{\Gamma}_{2}\right)=\widehat{D}_{n p f}\left(\widehat{\Gamma}_{2}, \widehat{\Gamma}_{1}\right)$;

H3. $\widehat{D}_{n p f}\left(\widehat{\Gamma}_{1}, \widehat{\Gamma}_{2}\right)=0$ iff $\widehat{\Gamma}_{1}=\widehat{\Gamma}_{2}$;

H4.If $\widehat{\Gamma}_{1} \subseteq \widehat{\Gamma}_{2} \subseteq \widehat{\Gamma}_{3}$, then $\widehat{D}_{n p f}\left(\widehat{\Gamma}_{1}, \widehat{\Gamma}_{3}\right) \geq \widehat{D}_{n p f}\left(\widehat{\Gamma}_{1}, \widehat{\Gamma}_{2}\right)$ and $\widehat{D}_{n p f}\left(\widehat{\Gamma}_{1}, \widehat{\Gamma}_{3}\right) \geq \widehat{D}_{n p f}\left(\widehat{\Gamma}_{2}, \widehat{\Gamma}_{3}\right)$.

Proof. H1: Since $0 \leq\left|\overline{\overline{P O}} \bar{\Gamma}_{1}-\overline{\overline{P O}} \bar{\Gamma}_{2}\right| \leq 1,0 \leq\left|\overline{\overline{N U}} \bar{\Gamma}_{1}-\overline{\overline{N U}} \bar{\Gamma}_{2}\right| \leq 1,0 \leq\left|\overline{\overline{N G}} \bar{\Gamma}_{1}-\overline{\overline{N G}} \widehat{\Gamma}_{2}\right| \leq 1$,

$$
\begin{aligned}
& 0 \leq\left|\max \left\{\overline{\overline{P O}} \bar{\Gamma}_{1}, \overline{\overline{N U}} \bar{\Gamma}_{2}\right\}-\max \left\{\overline{\overline{P O}} \bar{\Gamma}_{2}, \overline{\overline{N U}} \widehat{\Gamma}_{1}\right\}\right| \leq 1 \text { and } \\
& 0 \leq\left|\max \left\{\overline{\overline{P O}} \bar{\Gamma}_{1}, \overline{\overline{N G}} \bar{\Gamma}_{2}\right\}-\max \left\{\overline{\overline{P O}}_{\bar{\Gamma}_{2}}, \overline{\overline{N G}} \bar{\Gamma}_{1}\right\}\right| \leq 1,
\end{aligned}
$$

then we have

$$
\begin{aligned}
& 0 \leq\left(\frac { 1 } { 5 } \left(\left|\overline{\overline{P O}} \bar{\Gamma}_{1}-\overline{\overline{P O}} \bar{\Gamma}_{2}\right|^{\lambda}+\left|\overline{\overline{N U}} \bar{\Gamma}_{\Gamma_{1}}-\overline{\overline{N U}} \bar{\Gamma}_{2}\right|^{\lambda}+\left|\overline{\overline{N G}} \bar{\Gamma}_{1}-\overline{\overline{N G}} \bar{\Gamma}_{2}\right|^{\lambda}+\mid \max \left\{\overline{\overline{P O}} \bar{\Gamma}_{1}, \overline{\overline{N U}} \bar{\Gamma}_{2}\right\}-\right.\right. \\
& \left.\left.\max \left\{\overline{\overline{P O}} \bar{\Gamma}_{2}, \overline{\overline{N U}} \bar{\Gamma}_{1}\right\}\right|^{\lambda}+\left|\max \left\{\overline{\overline{P O}} \bar{\Gamma}_{1}, \overline{\overline{N G}} \bar{\Gamma}_{\Gamma_{2}}\right\}-\max \left\{\overline{\overline{P O}} \bar{\Gamma}_{2}, \overline{\overline{N G}} \bar{\Gamma}_{1}\right\}\right|^{\lambda}\right)\left.\right|^{1 / \lambda} . \leq 1 .
\end{aligned}
$$

Thus, $0 \leq \widehat{D}_{n p f}\left(\widehat{\Gamma}_{1}, \widehat{\Gamma}_{2}\right) \leq 1$.

H2: Clearly, we have $\widehat{D}_{n p f}\left(\widehat{\Gamma}_{1}, \widehat{\Gamma}_{2}\right)=\widehat{D}_{n p f}\left(\widehat{\Gamma}_{2}, \widehat{\Gamma}_{1}\right)$. 
H3: Necessity. If $\widehat{D}_{n p f}\left(\widehat{\Gamma}_{1}, \widehat{\Gamma}_{2}\right)=0$, then $\left|\overline{\overline{P O}} \widehat{\Gamma}_{1}-\overline{\overline{P O}}_{\Gamma_{2}}\right|=\left|\overline{\overline{N U}} \widehat{\Gamma}_{1}-\overline{\overline{N U}} \bar{\Gamma}_{2}\right|=\left|\overline{\overline{N G}} \widehat{\Gamma}_{1}-\overline{\overline{N G}} \widehat{\Gamma}_{2}\right|=$ $\left|\max \left\{\overline{\overline{P O}}_{\Gamma_{1}}\left(x_{i}\right), \overline{\overline{N U}}_{\Gamma_{2}}\left(x_{i}\right)\right\}-\max \left\{\overline{\overline{P O}}_{\bar{\Gamma}_{2}}, \overline{\overline{N U}} \bar{\Gamma}_{\Gamma_{1}}\right\}\right|=\left|\max \left\{\overline{\overline{P O}}_{\bar{\Gamma}_{1}}, \overline{\overline{N G}} \bar{\Gamma}_{2}\right\}-\max \left\{\overline{\overline{P O}} \bar{\Gamma}_{2}, \overline{\overline{N G}} \bar{\Gamma}_{1}\right\}\right|=0$, i.e., $\overline{\overline{P O}}_{\Gamma_{1}}=\overline{\overline{P O}}_{\Gamma_{2}}, \overline{\overline{N U}}_{\Gamma_{1}}=\overline{\overline{N U}}_{\Gamma_{2}}$ and $\overline{\overline{N G}} \widehat{\Gamma}_{1}=\overline{\overline{N G}}_{\Gamma_{2}}$. Thus, $\widehat{\Gamma}_{1}=\widehat{\Gamma}_{2}$ can be achieved.

Sufficiency. If $\widehat{\Gamma}_{1}=\widehat{\Gamma}_{2}$, then we have $\overline{\overline{P O}} \bar{\Gamma}_{1}=\overline{\overline{P O}} \bar{\Gamma}_{2}, \overline{\overline{N U}} \widehat{\Gamma}_{1}=\overline{\overline{N U}} \bar{\Gamma}_{2}$ and $\overline{\overline{N G}} \bar{\Gamma}_{1}=\overline{\overline{N G}} \bar{\Gamma}_{2}$, i.e., $\left|\overline{\overline{P O}} \bar{\Gamma}_{1}-\overline{\overline{P O}} \widehat{\Gamma}_{2}\right|=\left|\overline{\overline{N U}} \bar{\Gamma}_{1}-\overline{\overline{N U}} \widehat{\Gamma}_{2}\right|=\left|\overline{\overline{N G}} \bar{\Gamma}_{1}-\overline{\overline{N G}} \widehat{\Gamma}_{2}\right|=\mid \max \left\{\overline{\overline{P O}} \bar{\Gamma}_{1}, \overline{\overline{N U}} \bar{\Gamma}_{2}\right\}-$ $\max \left\{\overline{\overline{P O}}_{\Gamma_{2}}, \overline{\overline{N U}} \bar{\Gamma}_{1}\right\}|=| \max \left\{\overline{\overline{P O}}_{\bar{\Gamma}_{1}}, \overline{\overline{N G}} \bar{\Gamma}_{2}\right\}-\max \left\{\overline{\overline{P O}}_{\bar{\Gamma}_{2}}, \overline{\overline{N G}} \bar{\Gamma}_{1}\right\} \mid=0$.

Therefore, $\widehat{D}_{n p f}\left(\widehat{\Gamma}_{1}, \widehat{\Gamma}_{2}\right)=0$.

H4: If $\widehat{\Gamma}_{1} \subseteq \widehat{\Gamma}_{2} \subseteq \widehat{\Gamma}_{3}$, then we have $0 \leq \overline{\overline{P O}} \bar{\Gamma}_{1} \leq \overline{\overline{P O}} \bar{\Gamma}_{2} \leq \overline{\overline{P O}} \bar{\Gamma}_{3} \leq 1$, $0 \leq \overline{\overline{N U}} \bar{\Gamma}_{3} \leq \overline{\overline{N U}} \bar{\Gamma}_{2} \leq \overline{\overline{N U}} \widehat{\Gamma}_{1} \leq 1$ and $0 \leq \overline{\overline{N G}} \bar{\Gamma}_{3} \leq \overline{\overline{N G}} \widehat{\Gamma}_{2} \leq \overline{\overline{N G}} \widehat{\Gamma}_{1} \leq 1$.

Thus, $\left|\overline{\overline{P O}} \bar{\Gamma}_{1}-\overline{\overline{P O}} \widehat{\Gamma}_{2}\right|^{\lambda} \leq\left|\overline{\overline{P O}} \bar{\Gamma}_{1}-\overline{\overline{P O}} \bar{\Gamma}_{3}\right|^{\lambda},\left|\overline{\overline{N U}} \bar{\Gamma}_{1}-\overline{\overline{N U}} \bar{\Gamma}_{2}\right|^{\lambda} \leq\left|\overline{\overline{N U}} \bar{\Gamma}_{1}-\overline{\overline{N U}} \widehat{\Gamma}_{3}\right|^{\lambda}$, $\left|\overline{\overline{N G}} \widehat{\Gamma}_{1}-\overline{\overline{N G}} \widehat{\Gamma}_{2}\right|^{\lambda} \leq\left|\overline{\overline{N G}} \widehat{\Gamma}_{1}-\overline{\overline{N G}} \bar{\Gamma}_{3}\right|^{\lambda}$, and $1 \geq \max \left\{\overline{\overline{P O}}_{\Gamma_{3}}, \overline{\overline{N U}}{ }_{\Gamma_{1}}\right\} \geq \max \left\{\overline{\overline{P O}}_{\bar{\Gamma}_{2}}, \overline{\overline{N U}}{ }_{\Gamma_{1}}\right\} \geq \max \left\{\overline{\overline{P O}}_{\Gamma_{1}}, \overline{\overline{N U}}{\overline{\Gamma_{2}}}_{2}\right\} \geq \max \left\{\overline{\overline{P O}}{\overline{\Gamma_{1}}}_{\overline{N U}} \overline{\bar{\Gamma}}_{3}\right\} \geq 0$, i.e., $\left|\max \left\{\overline{\overline{P O}} \bar{\Gamma}_{1}, \overline{\overline{N U}}{ }_{\Gamma_{2}}\right\}-\max \left\{\overline{\overline{P O}} \bar{\Gamma}_{2}, \overline{\overline{N U}}{ }_{\Gamma_{1}}\right\}\right|^{\lambda} \leq\left|\max \left\{\overline{\overline{P O}} \bar{\Gamma}_{1}, \overline{\overline{N U}} \widehat{\Gamma}_{3}\right\}-\max \left\{\overline{\overline{P O}} \bar{\Gamma}_{3}, \overline{\overline{N U}} \bar{\Gamma}_{1}\right\}\right|^{\lambda}$ and $1 \geq \max \left\{\overline{\overline{P O}}_{\Gamma_{3}}, \overline{\overline{N G}} \bar{\Gamma}_{1}\right\} \geq \max \left\{\overline{\overline{P O}}_{\bar{\Gamma}_{2}}, \overline{\overline{N G}} \bar{\Gamma}_{1}\right\} \geq \max \left\{\overline{\overline{P O}}_{\Gamma_{1}}, \overline{\overline{N G}} \bar{\Gamma}_{2}\right\} \geq \max \left\{\overline{\overline{P O}}_{\bar{\Gamma}_{1}}, \overline{\overline{N G}} \bar{\Gamma}_{3}\right\} \geq 0$, i.e., $\left|\max \left\{\overline{\overline{P O}} \widehat{\Gamma}_{1}, \overline{\overline{N G}} \widehat{\Gamma}_{2}\right\}-\max \left\{\overline{\overline{P O}} \widehat{\Gamma}_{2}, \overline{\overline{N G}} \widehat{\Gamma}_{1}\right\}\right|^{\lambda} \leq\left|\max \left\{\overline{\overline{P O}} \widehat{\Gamma}_{1}, \overline{\overline{N G}} \widehat{\Gamma}_{3}\right\}-\max \left\{\overline{\overline{P O}} \widehat{\Gamma}_{2}, \overline{\overline{N G}} \widehat{\Gamma}_{1}\right\}\right|^{\lambda}$. Hence, $\widehat{D}_{n p f}\left(\widehat{\Gamma}_{1}, \widehat{\Gamma}_{3}\right) \geq \widehat{D}_{n p f}\left(\widehat{\Gamma}_{1}, \widehat{\Gamma}_{2}\right)$.

Similarly, $\widehat{D}_{n p f}\left(\widehat{\Gamma}_{1}, \widehat{\Gamma}_{3}\right) \geq \widehat{D}_{n p f}\left(\widehat{\Gamma}_{2}, \widehat{\Gamma}_{3}\right)$ can be obtained.

Property 1. Let $\widehat{\Gamma}_{1}$ and $\widehat{\Gamma}_{2}$ be two PFNs, then we have $\widehat{D}_{n p f}\left(\widehat{\Gamma}_{1}^{c}, \widehat{\Gamma}_{2}^{c}\right)=\widehat{D}_{n p f}\left(\widehat{\Gamma}_{1}, \widehat{\Gamma}_{2}\right)$, if $\overline{\overline{P O}}_{\Gamma_{1}}=\overline{\overline{N G}} \widehat{\Gamma}_{1}$ and $\overline{\overline{P O}} \widehat{\Gamma}_{2}=\overline{\overline{N G}} \bar{\Gamma}_{2}$.

Proof. Since $\widehat{\Gamma}_{1}^{c}=\left(\overline{\overline{N G}}_{\Gamma_{1}}, \overline{\overline{N U}}_{\Gamma_{1}}, \overline{\overline{P O}}_{\Gamma_{1}}\right)$ and $\widehat{\Gamma}_{2}^{c}=\left(\overline{\overline{N G}}_{\Gamma_{2}}, \overline{\overline{N U}} \bar{\Gamma}_{2}, \overline{\overline{P O}} \bar{\Gamma}_{2}\right)$, then $\widehat{D}_{n p f}\left(\widehat{\Gamma}_{1}^{c}, \widehat{\Gamma}_{2}^{c}\right)=\left(\frac{1}{5}\left(\left|\overline{\overline{N G}} \bar{\Gamma}_{1}-\overline{\overline{N G}} \widehat{\Gamma}_{2}\right|^{\lambda}+\left|\overline{\overline{N U}} \bar{\Gamma}_{1}\left(x_{i}\right)-\overline{\overline{N U}} \bar{\Gamma}_{2}\right|^{\lambda}+\left|\overline{\overline{P O}} \bar{\Gamma}_{1}-\overline{\overline{P O}} \bar{\Gamma}_{2}\right|^{\lambda}+\right.\right.$ $\left.\left|\max \left\{\overline{\overline{N G}} \bar{\Gamma}_{1}, \overline{\overline{N U}} \widehat{\Gamma}_{2}\right\}-\max \left\{\overline{\overline{N G}} \bar{\Gamma}_{2}, \overline{\overline{N U}} \bar{\Gamma}_{1}\right\}\right|^{\lambda}+\left|\max \left\{\overline{\overline{N G}} \bar{\Gamma}_{1}, \overline{\overline{P O}} \bar{\Gamma}_{2}\right\}-\max \left\{\overline{\overline{N G}} \bar{\Gamma}_{2}, \overline{\overline{P O}} \bar{\Gamma}_{1}\right\}\right|^{\lambda}\right)\left.\right|^{1 / \lambda}$. If $\overline{\overline{P O}} \bar{\Gamma}_{1}=\overline{\overline{N G}} \widehat{\Gamma}_{1}$ and $\overline{\overline{P O}} \widehat{\Gamma}_{2}=\overline{\overline{N G}} \widehat{\Gamma}_{2}$, then

$\widehat{D}_{n p f}\left(\widehat{\Gamma}_{1}^{c}, \widehat{\Gamma}_{2}^{c}\right)=\left(\frac{1}{5}\left(\left|\overline{\overline{N G}} \bar{\Gamma}_{1}-\overline{\overline{N G}}_{\Gamma_{2}}\right|^{\lambda}+\left|\overline{\overline{N U}} \bar{\Gamma}_{1}-\overline{\overline{N U}}_{\bar{\Gamma}_{2}}\right|^{\lambda}+\left|\overline{\overline{P O}} \bar{\Gamma}_{1}-\overline{\overline{P O}}_{\bar{\Gamma}_{2}}\right|^{\lambda}+\right.\right.$ 


$$
\begin{aligned}
& \left|\max \left\{\overline{\overline{P O}} \bar{\Gamma}_{1}, \overline{\overline{N U}} \widehat{\Gamma}_{2}\right\}-\max \left\{\overline{\overline{P O}} \bar{\Gamma}_{2}, \overline{\overline{N U}} \widehat{\Gamma}_{1}\right\}\right|^{\lambda}+\mid \max \left\{\overline{\overline{P O}} \bar{\Gamma}_{1}, \overline{\overline{N G}} \widehat{\Gamma}_{2}\right\}- \\
& \left.\left.\max \left\{\overline{\overline{P O}} \bar{\Gamma}_{2}, \overline{\overline{N G}} \bar{\Gamma}_{1}\right\}\right|^{\lambda}\right)\left.\right|^{1 / \lambda}=\widehat{D}_{n p f}\left(\widehat{\Gamma}_{1}, \widehat{\Gamma}_{2}\right) .
\end{aligned}
$$

Property 2. Let $\widehat{\Gamma}_{1}$ and $\widehat{\Gamma}_{2}$ be two PFNs, then the following results can be achieved:

(1) If $\widehat{\Gamma}_{1} \subseteq \widehat{\Gamma}_{2}$, then $\widehat{D}_{n p f}\left(\widehat{\Gamma}_{1} \cup \widehat{\Gamma}_{2}, \widehat{\Gamma}_{1} \cap \widehat{\Gamma}_{2}\right)=\widehat{D}_{n p f}\left(\widehat{\Gamma}_{1}, \widehat{\Gamma}_{2}\right)$;

(2) If $\widehat{\Gamma}_{1} \supseteq \widehat{\Gamma}_{2}$, then $\widehat{D}_{n p f}\left(\widehat{\Gamma}_{1} \cap \widehat{\Gamma}_{2}, \widehat{\Gamma}_{1} \cup \widehat{\Gamma}_{2}\right)=\widehat{D}_{n p f}\left(\widehat{\Gamma}_{1}, \widehat{\Gamma}_{2}\right)$.

Proof. (1) If $\widehat{\Gamma}_{1} \subseteq \widehat{\Gamma}_{2}$, then we have $\overline{\overline{P O}} \bar{\Gamma}_{1} \leq \overline{\overline{P O}} \bar{\Gamma}_{2}, \overline{\overline{N U}} \bar{\Gamma}_{1} \geq \overline{\overline{N U}} \bar{\Gamma}_{2}$ and $\overline{\overline{N G}} \widehat{\Gamma}_{1} \geq \overline{\overline{N G}} \bar{\Gamma}_{2}$.

Based on the operations in Definition 2, the following results are achieved:

$$
\begin{aligned}
& \left|\overline{\overline{P O}} \bar{\Gamma}_{1} \cup \widehat{\Gamma}_{2}-\overline{\overline{P O}}_{\bar{\Gamma}_{1} \cap \widehat{\Gamma}_{2}}\right|=\left|\max \left\{\overline{\overline{P O}}_{\bar{\Gamma}_{1}}, \overline{\overline{P O}} \bar{\Gamma}_{2}\right\}-\min \left\{\overline{\overline{P O}}_{\bar{\Gamma}_{1}}, \overline{\overline{P O}} \bar{\Gamma}_{2}\right\}\right|=\left|\overline{\overline{P O}} \bar{\Gamma}_{2}-\overline{\overline{P O}} \bar{\Gamma}_{1}\right| ; \\
& \left|\overline{\overline{N U}} \bar{\Gamma}_{1} \cup \widehat{\Gamma}_{2}-\overline{\overline{N U}} \bar{\Gamma}_{1} \cap \widehat{\Gamma}_{2}\right|=\left|\min \left\{\overline{\overline{N U}} \bar{\Gamma}_{1}, \overline{\overline{N U}} \bar{\Gamma}_{2}\right\}-\max \left\{\overline{\overline{N U}} \bar{\Gamma}_{1}, \overline{\overline{N U}} \bar{\Gamma}_{2}\right\}\right|=\left|\overline{\overline{N U}} \bar{\Gamma}_{1}-\overline{\overline{N U}} \widehat{\Gamma}_{2}\right| ; \\
& \left|\overline{\overline{N G}} \widehat{\Gamma}_{1} \cup \widehat{\Gamma}_{2}-\overline{\overline{N G}} \widehat{\Gamma}_{1} \cap \widehat{\Gamma}_{2}\right|=\left|\min \left\{\overline{\overline{N G}} \bar{\Gamma}_{1}, \overline{\overline{N G}} \bar{\Gamma}_{2}\right\}-\max \left\{\overline{\overline{N G}} \bar{\Gamma}_{1}, \overline{\overline{N G}} \bar{\Gamma}_{2}\right\}\right|=\left|\overline{\overline{N G}} \bar{\Gamma}_{2}-\overline{\overline{N G}} \bar{\Gamma}_{1}\right| ; \\
& \left|\max \left\{\overline{\overline{P O}} \bar{\Gamma}_{1} \cup \widehat{\Gamma}_{2}, \overline{\overline{N U}} \bar{\Gamma}_{1} \cap \widehat{\Gamma}_{2}\right\}-\max \left\{\overline{\overline{P O}} \bar{\Gamma}_{1} \cap \widehat{\Gamma}_{2}, \overline{\overline{N U}} \bar{\Gamma}_{1} \cup \widehat{\Gamma}_{2}\right\}\right|=\mid \max \left\{\max \left\{\overline{\overline{P O}} \bar{\Gamma}_{1}, \overline{P S} \bar{\Gamma}_{2}\right\},\right. \\
& \left.\max \left\{\overline{\overline{N U}} \bar{\Gamma}_{1}, \overline{\overline{N U}} \bar{\Gamma}_{2}\right\}\right\}-\max \left\{\min \left\{\overline{\overline{P O}}_{\bar{\Gamma}_{1}}, \overline{\overline{P S}} \bar{\Gamma}_{2}\right\}, \min \left\{\overline{\overline{N U}} \bar{\Gamma}_{1}, \overline{\overline{N U}} \widehat{\Gamma}_{2}\right\}\right\}= \\
& \left|\max \left\{\overline{\overline{P O}} \bar{\Gamma}_{2}, \overline{\overline{N U}} \widehat{\Gamma}_{1}\right\}-\max \left\{\overline{\overline{P O}} \bar{\Gamma}_{1}, \overline{\overline{N U}} \widehat{\Gamma}_{2}\right\}\right| ; \\
& \left|\max \left\{\overline{\overline{P O}} \bar{\Gamma}_{1} \cup \widehat{\Gamma}_{2}, \overline{\overline{N G}} \bar{\Gamma}_{1} \cap \widehat{\Gamma}_{2}\right\}-\max \left\{\overline{\overline{P O}} \bar{\Gamma}_{1} \cap \widehat{\Gamma}_{2}, \overline{\overline{N G}} \bar{\Gamma}_{1} \cup \widehat{\Gamma}_{2}\right\}\right|=\mid \max \left\{\max \left\{\overline{\overline{P O}} \bar{\Gamma}_{1}, \overline{\overline{P S}} \widehat{\Gamma}_{2}\right\},\right. \\
& \left.\max \left\{\overline{\overline{N G}}_{\Gamma_{1}}, \overline{\overline{N G}} \bar{\Gamma}_{2}\right\}\right\}-\max \left\{\min \left\{\overline{\overline{P O}}_{\bar{\Gamma}_{1}}, \overline{\overline{P O}}_{\bar{\Gamma}_{2}}\right\}, \min \left\{\overline{\overline{N G}}_{\bar{\Gamma}_{1}}, \overline{\overline{N G}} \bar{\Gamma}_{2}\right\}\right\}= \\
& \left|\max \left\{\overline{\overline{P O}} \bar{\Gamma}_{2}, \overline{\overline{N G}} \bar{\Gamma}_{1}\right\}-\max \left\{\overline{\overline{P O}} \bar{\Gamma}_{1}, \overline{\overline{N G}} \bar{\Gamma}_{2}\right\}\right| \text {. }
\end{aligned}
$$

Therefore, $\widehat{D}_{n p f}\left(\widehat{\Gamma}_{1} \cup \widehat{\Gamma}_{2}, \widehat{\Gamma}_{1} \cap \widehat{\Gamma}_{2}\right)=\widehat{D}_{n p f}\left(\widehat{\Gamma}_{1}, \widehat{\Gamma}_{2}\right)$.

(2) Similarly, if $\widehat{\Gamma}_{1} \supseteq \widehat{\Gamma}_{2}$, then $\widehat{D}_{n p f}\left(\widehat{\Gamma}_{1} \cap \widehat{\Gamma}_{2}, \widehat{\Gamma}_{1} \cup \widehat{\Gamma}_{2}\right)=\widehat{D}_{n p f}\left(\widehat{\Gamma}_{1}, \widehat{\Gamma}_{2}\right)$ can be obtained.

\section{An integrated ANP-TODIM approach based on a novel normalized picture fuzzy distance measure}

Assume $C=\left\{c_{1}, c_{2}, \ldots, c_{m}\right\}$ denotes the set of criteria and $\Phi=\left\{\alpha_{1}, \alpha_{2}, \ldots, \alpha_{n}\right\}$ denotes the group of alternatives. $\alpha_{i j}=\left(\overline{\overline{P O}}_{\alpha_{i j}}, \overline{\overline{\overline{N U}}} \alpha_{i j}, \overline{\overline{N G}} \alpha_{i j}\right)$ represents the assessment value of $\alpha_{i}$ under criterion $c_{j}$, where $\overline{\overline{P O}}_{\alpha_{i j}}$ and $\overline{\overline{N U}}_{\alpha_{i j}}$ indicate the satisfaction and neutrality, respectively, in which the alternative $\alpha_{i}$ under the criterion $c_{j}$, while $\overline{\overline{N G}}_{\alpha_{i j}}$ represents the dissatisfaction alternative $\alpha_{i}$ under the criterion $c_{j}$. 


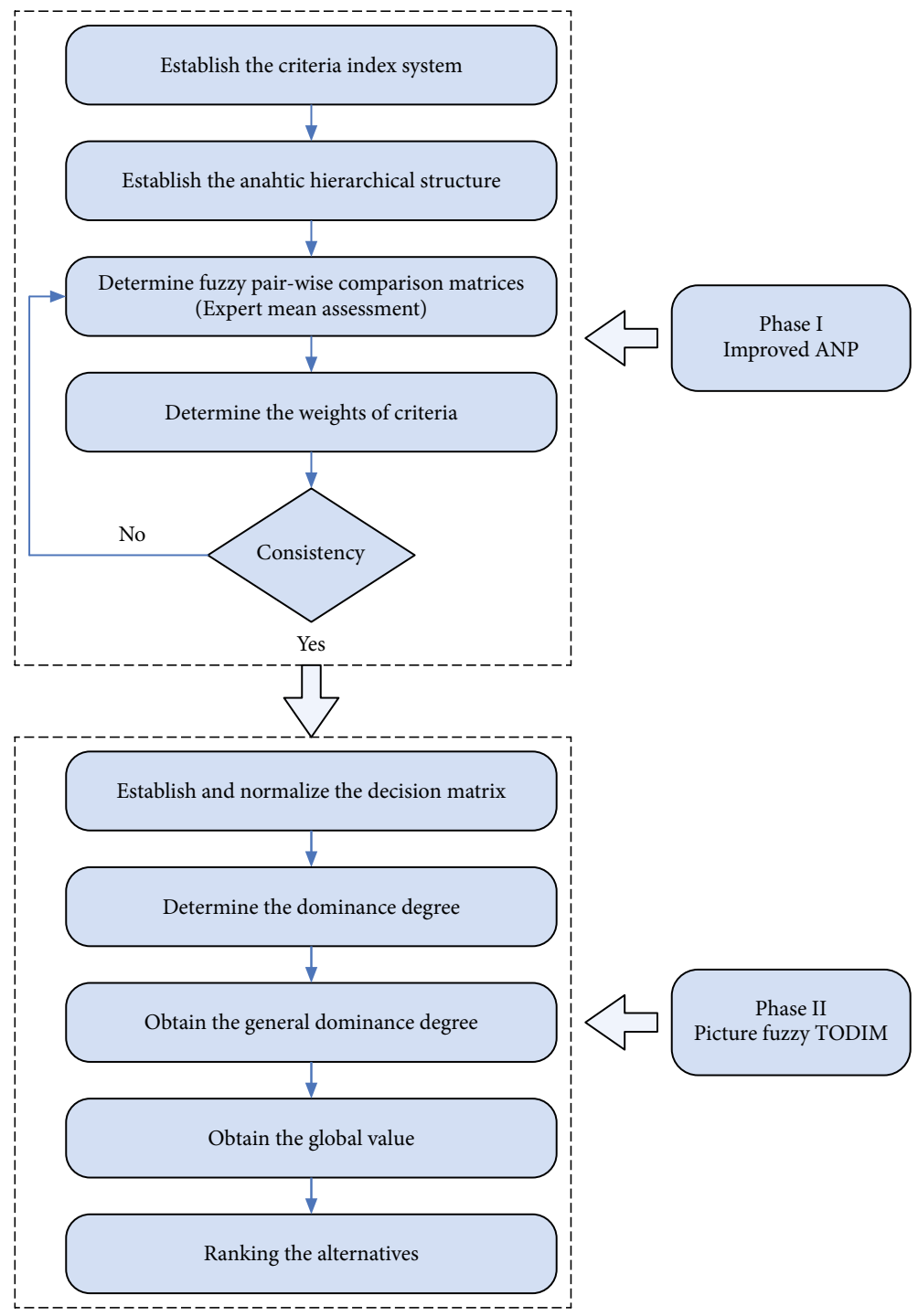

Figure 1. Illustration of the flowchart of the method

Figure 1 shows the flowchart of the method in this paper. The process to choose the optimal alternative(s) is provided based on ANP-TODIM approach in the following steps.

\section{Phase 1. Determine the weights of criteria using an improved ANP method}

In this phase, an improved ANP method is utilized to obtain the weight vectors. First, the multi-criteria index system and analytic hierarchical structure for MCDM problems are established according to the relationships between the influencing factors. A judgment matrix is then created, and we obtained the weight of each criterion by using Super Decisions software. 
Step 1.1. Establish the criteria index system. A multi-criteria index system for MCDM problems can be established and evaluated by experts, academicians, and corresponding associates via relevant literature review.

Step 1.2. Establish the analytic hierarchical structure. The analytic hierarchical structure can be established by using a goal layer, criteria layer, sub-criteria layer, and alternatives layer.

Step 1.3. Determine fuzzy pair-wise comparison matrices. To develop comparison matrices, experts should compare the importance of two criteria according to the scale presented in Table 1. However, these experts may exhibit subjectivity when the relative importance of the two criteria is determined, leading to reduce the reliability of the weighting of these criteria. Therefore, we suggest an expert mean assessment method to improve the traditional ANP, avoiding errors caused by human factors (Zhang, Wang, \& Yang, 2018). Firstly, experts judge the relative importance of two criteria according to the fuzzy linguistic scale, as shown in Table 1. Next, the mean values of all experts' scores are calculated, in addition to the deviations between each expert's score and the mean values. If all deviations fall within the confidence region, then the mean value gives the final relative importance of the two criteria. However, if the deviation exceeds the confidence region, then the corresponding expert's score should be deleted. In the latter case, the mean value of the remaining experts' scores should be recalculated, and the steps are repeated until all the deviations fall within the confidence region. The key part of the expert mean assessment is to determine the confidence region. Since the maximum crisp value of linguistic scale presented in Table 1 is 9 , thus the median value can be taken as the confidence interval $[-4.5,4.5]$ (Barton, Nelson, \& Xie, 2014). However, since the selected experts have the experience in their domain, then there will be generally no major mistakes. Therefore, their confidence interval can be halved, i.e., $[-2.3,2.3]$ can be determined as final confidence interval (Chen \& Tang, 2014). Finally, matrices can be constructed.

The consistency of each pair-wise comparison matrices should be examined as described in Figure 1. If the consistency ratio is greater than 0.1 , then consistency cannot be satisfied. The pair-wise comparison matrices should be accordingly revised. Step 1.3 should be then repeated as well.

Table 1. A fuzzy linguistic scale using an improved ANP

\begin{tabular}{|l|l|}
\hline Linguistic Term & Crisp Value \\
\hline Exactly equal important & 1 \\
\hline Average important & 3 \\
\hline High important & 5 \\
\hline Very high important & 7 \\
\hline Certainly high important & 9 \\
\hline Intermediate value & $2,4,6,8$ \\
\hline
\end{tabular}

Step 1.4. Determine the weights of the criteria. According to the pair-wise comparison matrices determined in Step 1.3, the weight of criteria can be obtained by using Super Decisions software. 


\section{Phase II. The extended picture fuzzy TODIM method}

Step 2.1. Establish and normalize the decision matrix. According to a DMs' knowledge and experience, the experts invited in phase 1 should provide evaluation values for criteria of each alternative in form of PFNs $\alpha_{i j}(i=1,2, \ldots, n ; j=1,2, \ldots, m)$. After that, the decision matrix can be constructed.

As the most common criteria for decision-making problems involve costs and benefits, thus the evaluation value $\alpha_{i j}$ should be normalized to $\tilde{\alpha}_{i j}$ to unify all criteria. For the benefit criteria, $\tilde{\alpha}_{i j}=\alpha_{i j}$, while for the cost criteria, the evaluation value should be normalized as $\tilde{\alpha}_{i j}=\alpha_{i j}^{c}$. Here, $\alpha_{i j}^{c}$ has been already provided in Definition 2.

Step 2.2. Determine the dominance degree. From the following mathematical formula, the dominance degree of the alternative $\alpha_{i}$ over the alternative $\alpha_{k}$ concerning the criterion $c_{j}$ can be determined as follows:

$$
\psi_{j}\left(\alpha_{i}, \alpha_{k}\right)=\left\{\begin{array}{l}
\sqrt{\widehat{D}_{n p f}\left(\alpha_{i j}, \alpha_{k j}\right) \varpi_{l j} /\left(\sum_{j=1}^{n} \varpi_{l j}\right)}, \quad \alpha_{k j} \prec \alpha_{i j} \\
0, \\
-\frac{1}{\theta} \sqrt{\widehat{D}_{n p f}\left(\alpha_{i j}, \alpha_{k j}\right)\left(\sum_{j=1}^{n} \varpi_{l j}\right) / \varpi_{l j},} \quad \alpha_{i j} \prec \alpha_{k j}
\end{array},\right.
$$

where $\hat{D}_{n p f}\left(\alpha_{i j}, \alpha_{k j}\right)$ denotes the novel normalized picture fuzzy distance between $\alpha_{i j}$ and $\alpha_{k j}$. Besides, $\varpi_{l j}=\varpi_{j} / \varpi_{l}(j=1,2, \ldots, m)$ represents the relative weight of corresponding criterion $c_{j}$ under reference criterion $c_{l}$, and $\varpi_{l}=\max \left\{\varpi_{j} \mid j=1,2, \ldots, m\right\}$. In addition, $\theta$ denotes the loss aversion coefficient. Different $\theta$ can result in different shapes of the prospect theoretical value function in the negative quadrant. The higher the value of $\theta$, the higher the loss aversion of DMs.

Step 2.3. Obtain the general dominance degree. The general dominance degree of $\alpha_{i}$ with regard to $\alpha_{k}$ is determined as follows:

$$
\varsigma\left(\alpha_{i}, \alpha_{k}\right)=\sum_{j=1}^{m} \psi_{j}\left(\alpha_{i}, \alpha_{k}\right) .
$$

Step 2.4. Obtain the global value. Based on Step 2.3, the global value of alternative $\xi\left(\alpha_{i}\right)$ of $\alpha_{i}$ can be calculated as:

$$
\xi\left(\alpha_{i}\right)=\frac{\sum_{k=1}^{n} \varsigma\left(\alpha_{i}, \alpha_{k}\right)-\min _{i \in m}\left\{\sum_{k=1}^{n} \varsigma\left(\alpha_{i}, \alpha_{k}\right)\right\}}{\max _{i \in m}\left\{\sum_{k=1}^{n} \varsigma\left(\alpha_{i}, \alpha_{k}\right)\right\}-\min _{i \in m}\left\{\sum_{k=1}^{n} \varsigma\left(\alpha_{i}, \alpha_{k}\right)\right\}} .
$$

Step 2.5. Rank the alternatives. The alternatives can be ranked in descending order. The greater the value of $\xi\left(\alpha_{i}\right)$, the better the alternative $\alpha_{i}$ should be. 


\section{An illustrative example}

An example taken from Wen, Shi, Cai, Miao, and Long (2014) is demonstrated, which verifies the efficiency of the method in this paper. With the widespread popularity of the mobile Internet and increasing demand of tourists in terms of the quality of tourism services, online travel, mobile travel, and other services have gradually popularized. Due to information overload, personalized travel recommendation is being highly important. Personalized tourism recommendation can meet the requirements in their mind, and help him/her make travel decisions more quickly. In a personalized tourism recommendation system, it mainly includes four criteria (the tourist's travel demand, tourist's preference for tourism products, any constraints, and tourism resource bank), in which information is utilized to make accurate and effective recommendations. According to these four criteria, tourists should select the best tourism attractions on the basis of their own preferences and the actual location of the candidate tourism attractions. Suppose five tourism attractions, $\alpha_{1}, \alpha_{2}, \alpha_{3}, \alpha_{4}, \alpha_{5}$, are taken into consideration. During assessment, four criteria are considered, namely: $c_{1}$ : tourist's travel demand; $c_{2}$ : tourist's preference; $c_{3}$ : constraints; and $c_{4}$ : tourism resource bank. The corresponding weights of the four criteria are fully unknown. The five candidates $\alpha_{i}(i=1,2,3,4,5)$ can be assessed by using PFNs with a DM under the criterion $\mathrm{c}_{k}(k=1,2,3,4)$.

\subsection{Apply the ANP-TODIM method to tourism attraction recommendation}

The steps to select the most optimal tourism attraction recommendation based on the ANPTODIM approach is presented in the following.

\section{Phase 1. Determine the weights for criteria using an improved ANP}

Step 1.1. Establish the criteria for tourism attraction recommendation. In this phase, twenty experts were consulted, which included tourists, academicians, and tourism associates. Based on their suggestions, plus relevant literature reviews, four criteria and twenty-one sub-criteria were identified to evaluate the tourism attraction recommendation as presented in Table 2.

Table 2. Criteria for tourism attraction recommendation

\begin{tabular}{|c|c|c|}
\hline Criteria & Sub-criteria & Remark \\
\hline $\begin{array}{l}\text { (C1) Tourist's travel } \\
\text { demand }\end{array}$ & $\begin{array}{l}\text { (C11) The user's travel days } \\
\text { (C12) Consumer spending } \\
\text { (C13) Departure date } \\
\text { (C14) Hotel environment }\end{array}$ & $\begin{array}{l}\text { Tourism demand refers to the user's } \\
\text { demand for the tourism products that the } \\
\text { user wants to buy, which is a subjective } \\
\text { condition }\end{array}$ \\
\hline $\begin{array}{l}\text { (C2) Tourist's } \\
\text { preference }\end{array}$ & $\begin{array}{l}\text { (C21) Food preference } \\
\text { (C22) Residence preference } \\
\text { (C23) Traffic preference } \\
\text { (C24) Entertainment preference } \\
\text { (C25) Purchase preference } \\
\text { (C26) Attractions preference }\end{array}$ & $\begin{array}{l}\text { The tourist's preference for tourism } \\
\text { products can be obtained by analyzing } \\
\text { factors such as the tourist's historical } \\
\text { travel data (such as the tourist's photos } \\
\text { uploaded by the user and the user's } \\
\text { comments about the tourism products), } \\
\text { the pages on the travel website and } \\
\text { purchase records of tourism products }\end{array}$ \\
\hline
\end{tabular}


End of Table 2

\begin{tabular}{|c|c|c|}
\hline Criteria & Sub-criteria & Remark \\
\hline (C3) Constraints & $\begin{array}{l}\text { (C31) The age or gender } \\
\text { of tourist } \\
\text { (C32) The physical condition } \\
\text { of the tourist } \\
\text { (C33) The income of the tourist } \\
\text { (C34) Weather conditions at the } \\
\text { tourism attractions } \\
\text { (C35) Accompanied by children } \\
\text { and the elderly or not }\end{array}$ & $\begin{array}{l}\text { Constraints are described as some } \\
\text { objective conditions that affect the user's } \\
\text { decision making about tourism }\end{array}$ \\
\hline $\begin{array}{l}\text { (C4) Tourism } \\
\text { resource bank }\end{array}$ & $\begin{array}{l}\text { (C41) Food } \\
\text { (C42) Residence } \\
\text { (C43) Traffic } \\
\text { (C44) Entertainment } \\
\text { (C45) Purchase } \\
\text { (C46) Tourism attractions }\end{array}$ & $\begin{array}{l}\text { The tourism resource bank contains six } \\
\text { aspects of the tourism products. It also } \\
\text { contains the attribute data of various } \\
\text { kinds of tourism products. The tourism } \\
\text { resource bank is the one of the most } \\
\text { important pieces of data to analyze }\end{array}$ \\
\hline
\end{tabular}

Step 1.2. Construct the ANP structure. According to the criteria presented in Table 2, the ANP structure of tourism attraction recommendation was constructed, as illustrated in Figure 2.

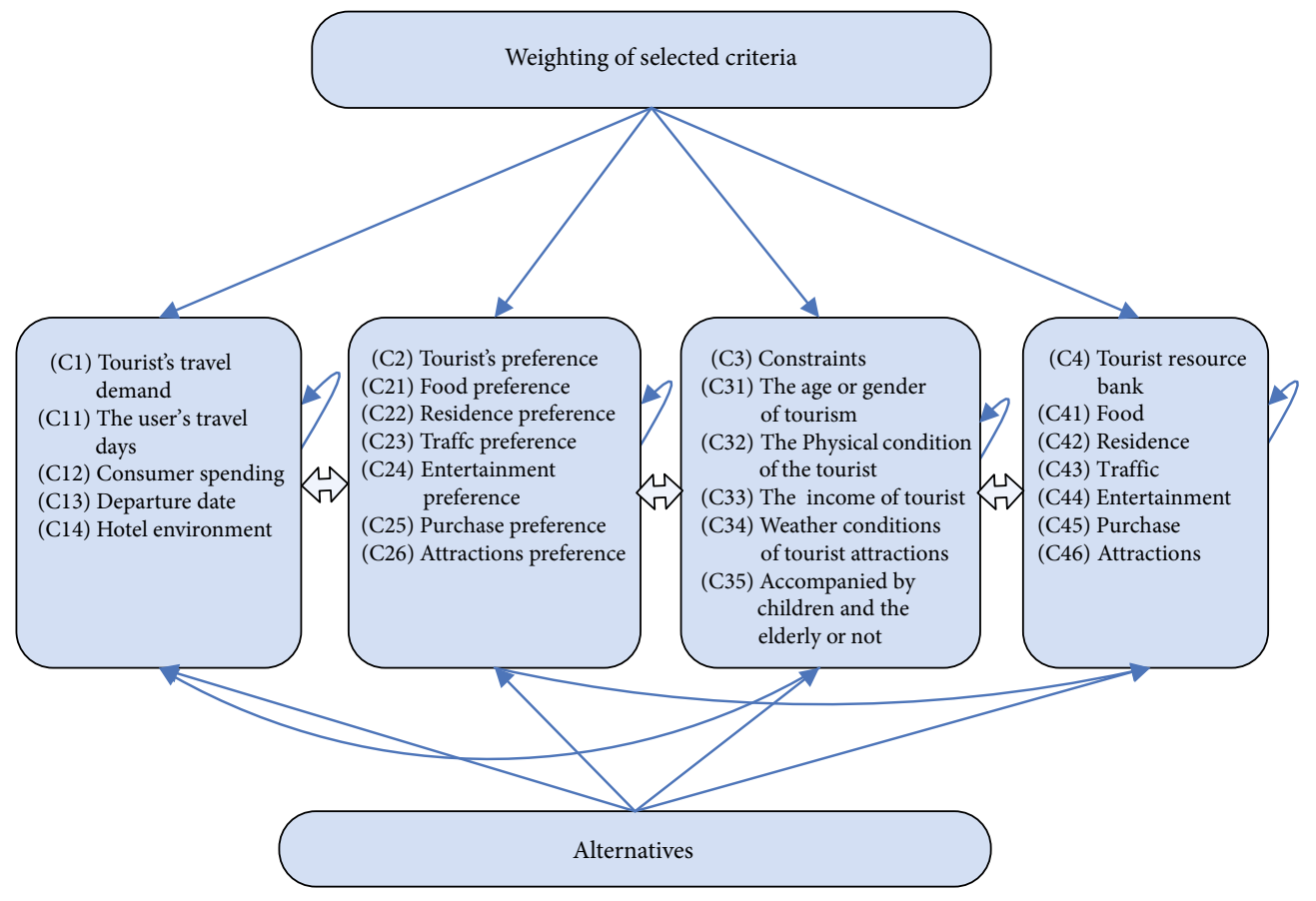

Figure 2. The ANP structure of the tourism attraction recommendation 
Step 1.3. Determine fuzzy pair-wise comparison matrices. To analyze all criteria and subcriteria, the experts invited in Phase 1 were consulted. The selected experts had in-depth knowledge about tourism, and concluded the level of importance of each sub-criterion, which were then utilized based on fuzzy linguistic scale (see Table 1), in order to construct the comparison matrices. The corresponding data were then analyzed using Super Decisions software based on the expert mean assessment method (Zhang et al., 2018). As the consistency ratios of all pair-wise comparison matrices did not exceed 0.1 , the pair-wise comparison matrices were accordingly valid.

Step 1.4. Calculate the weight vector. According to the results presented in Step 1.3, the weight distribution of criteria can be obtained by using Super Decisions software, as presented in Table 3.

Table 3. Weight distribution of criteria for tourism attraction recommendation

\begin{tabular}{|c|c|c|c|c|}
\hline Criteria & Sub-criteria & $\begin{array}{c}\text { Criteria } \\
\text { weight }\end{array}$ & $\begin{array}{c}\text { Relative } \\
\text { weight }\end{array}$ & $\begin{array}{l}\text { Comprehensive } \\
\text { weight }\end{array}$ \\
\hline \multirow{3}{*}{$\begin{array}{l}\text { (C1) Tourist's } \\
\text { travel demand }\end{array}$} & (C11) The user's travel days & 0.085 & 0.272 & \multirow{3}{*}{0.313} \\
\hline & (C12) Consumer spending & 0.152 & 0.486 & \\
\hline & (C13) Hotel environment & 0.076 & 0.243 & \\
\hline \multirow{6}{*}{$\begin{array}{l}\text { (C2) Tourist's } \\
\text { preference }\end{array}$} & (C21) Food preference & 0.033 & 0.169 & \multirow{6}{*}{0.195} \\
\hline & (C22) Residence preference & 0.044 & 0.226 & \\
\hline & (C23) Traffic preference & 0.039 & 0.200 & \\
\hline & (C24) Entertainment preference & 0.014 & 0.072 & \\
\hline & (C25) Purchase preference & 0.020 & 0.103 & \\
\hline & (C26) Attractions preference & 0.045 & 0.231 & \\
\hline \multirow{5}{*}{ (C3) Constraints } & (C31) The age or gender of the tourist & 0.034 & 0.157 & \multirow{5}{*}{0.217} \\
\hline & (C32) The physical condition of the tourist & 0.050 & 0.230 & \\
\hline & (C33) The income of the tourist & 0.041 & 0.189 & \\
\hline & $\begin{array}{l}\text { (C34) Weather conditions at the tourism } \\
\text { attractions }\end{array}$ & 0.039 & 0.180 & \\
\hline & $\begin{array}{l}\text { (C35) Accompanied by children and the } \\
\text { elderly or not }\end{array}$ & 0.053 & 0.244 & \\
\hline \multirow{6}{*}{$\begin{array}{l}\text { (C4) Tourism } \\
\text { resource bank }\end{array}$} & (C41) Food & 0.043 & 0.156 & \multirow{6}{*}{0.275} \\
\hline & (C42) Residence & 0.054 & 0.196 & \\
\hline & (C43) Traffic & 0.049 & 0.178 & \\
\hline & (C44) Entertainment & 0.034 & 0.124 & \\
\hline & (C45) Purchase & 0.030 & 0.109 & \\
\hline & (C46) Tourism attractions & 0.065 & 0.236 & \\
\hline
\end{tabular}

Thus, the weights of criteria are obtained as $\varpi=(0.313,0.195,0.217,0.275)^{T}$. 


\section{Phase II. An extended picture fuzzy TODIM method}

Step 2.1. Establish and normalize the decision matrix. The experts invited earlier can provide evaluation values about the four criteria based on their experience and knowledge in form of PFNs $\alpha_{i j}(i=1,2, \ldots, 5 ; j=1,2, \ldots, 4)$. In order to ensure the accuracy and validity of the evaluation information, there is no indication about any decision made during the evaluation process, and the experts are not allowed to communicate with each other. Then, the decision matrix, as outlined in Table 4, can be constructed. Moreover, all criteria are benefit type, and therefore are spared from normalization.

Table 4. The picture fuzzy decision matrix

\begin{tabular}{|c|c|c|c|c|}
\hline & $c_{1}$ & $c_{2}$ & $c_{3}$ & $c_{4}$ \\
\hline$\alpha_{1}$ & $(0.65,0.13,0.21)$ & $(0.53,0.13,0.25)$ & $(0.64,0.11,0.15)$ & $(0.66,0.09,0.18)$ \\
\hline$\alpha_{2}$ & $(0.60,0.11,0.19)$ & $(0.55,0.07,0.23)$ & $(0.58,0.09,0.22)$ & $(0.62,0.10,0.12)$ \\
\hline$\alpha_{3}$ & $(0.58,0.15,0.14)$ & $(0.61,0.12,0.17)$ & $(0.61,0.08,0.21)$ & $(0.64,0.16,0.05)$ \\
\hline$\alpha_{4}$ & $(0.52,0.11,0.21)$ & $(0.59,0.10,0.23)$ & $(0.55,0.14,0.2)$ & $(0.67,0.13,0.10)$ \\
\hline$\alpha_{5}$ & $(0.53,0.06,0.29)$ & $(0.62,0.07,0.22)$ & $(0.63,0.13,0.20)$ & $(0.64,0.06,0.22)$ \\
\hline
\end{tabular}

Step 2.2. Determine the dominance degree. According to Eq. (7), for the convenience of analysis and computation, the novel normalized picture fuzzy Hausdorff distance measure is used and $\theta=2.25$ and $\widehat{D}_{n p f h}\left(\alpha_{i j}, \alpha_{k j}\right)$ are obtained; i.e. $\lambda=1$. After that, the dominance degree concerning the criteria $c_{1}, c_{2}, c_{3}$ and $c_{4}$ can be respectively obtained as follows:

$$
\begin{aligned}
& \psi_{1}=\left[\begin{array}{ccccc}
0 & 0.109 & -0.195 & 0.160 & 0.179 \\
-0.155 & 0 & -0.138 & 0.128 & 0.150 \\
-0.195 & -0.138 & 0 & 0.135 & 0.156 \\
-0.228 & -0.181 & -0.191 & 0 & 0.100 \\
-0.254 & -0.213 & -0.222 & -0.142 & 0
\end{array}\right] \\
& \psi_{2}=\left[\begin{array}{ccccc}
0 & -0.168 & -0.259 & -0.216 & -0.270 \\
-0.168 & 0 & -0.242 & -0.174 & -0.211 \\
0.113 & 0.106 & 0 & 0.074 & -0.162 \\
-0.216 & 0.077 & -0.168 & 0 & -0.162 \\
-0.270 & 0.093 & -0.162 & -0.162 & 0
\end{array}\right] \text {; } \\
& \psi_{3}=\left[\begin{array}{ccccc}
0 & 0.108 & 0.088 & 0.123 & 0.066 \\
-0.222 & 0 & -0.142 & -0.171 & -0.196 \\
-0.181 & -0.142 & 0 & -0.213 & -0.148 \\
-0.252 & -0.171 & -0.213 & 0 & -0.213 \\
-0.135 & 0.096 & -0.148 & -0.213 & 0
\end{array}\right] \text {; } \\
& \psi_{4}=\left[\begin{array}{ccccc}
0 & -0.165 & -0.193 & -0.147 & 0.085 \\
0.102 & 0 & -0.165 & -0.170 & 0.105 \\
0.120 & 0.102 & 0 & -0.156 & 0.122 \\
0.091 & 0.105 & -0.156 & 0 & 0.124 \\
-0.137 & -0.170 & -0.197 & -0.201 & 0
\end{array}\right] \text {. }
\end{aligned}
$$


Step 2.3. Obtain the general dominance degree. From Eq. (8), the general dominance degree matrix can be constructed as follows:

$$
\varsigma=\left[\begin{array}{ccccc}
0 & -0.116 & -0.558 & -0.079 & 0.0591 \\
-0.443 & 0 & -0.687 & -0.387 & -0.152 \\
-0.143 & -0.071 & 0 & -0.161 & -0.032 \\
-0.605 & -0.171 & -0.729 & 0 & -0.151 \\
-0.795 & -0.195 & -0.729 & -0.718 & 0
\end{array}\right] .
$$

Step 2.4. Obtain the global value. From Eq. (9), the global value of the alternative $\alpha_{i}$ is determined as:

$$
\xi\left(\alpha_{1}\right)=0.858 ; \xi\left(\alpha_{2}\right)=0.379 ; \xi\left(\alpha_{3}\right)=1 ; \xi\left(\alpha_{4}\right)=0.385 ; \xi\left(\alpha_{5}\right)=0 .
$$

Step 2.5. Rank the alternatives. Based on Step 2.4, $\xi\left(\alpha_{5}\right)<\xi\left(\alpha_{2}\right)<\xi\left(\alpha_{4}\right)<\xi\left(\alpha_{1}\right)<\xi\left(\alpha_{3}\right)$ can be obtained; therefore, the ranking $\alpha_{5} \prec \alpha_{2} \prec \alpha_{4} \prec \alpha_{1} \prec \alpha_{3}$ is achieved. Thus, $\alpha_{3}$ is the best alternative.

\subsection{Sensitivity analysis}

In this sub-section, we investigated the influence of parameters $\theta$ and $\lambda$ on the final order of alternatives. For the first case, $\theta=2.25$ was fixed, and only the changes to the parameter $\lambda$ were considered, i.e., $1 \leq \lambda \leq 10$. For the second case, the changes to two parameters $\theta$ and $\lambda$ were simultaneously taken into account, i.e., $0<\theta \leq 5$ and $1 \leq \lambda \leq 10$. The results of the two cases are displayed in Figures 3-8.

Apparently, if $\theta=2.25$ and $1 \leq \lambda \leq 10$, then the same rankings can be obtained as $\lambda$ changes (see Table 5). Besides, the parameter $\lambda$ does not affect the final ranking of alternatives. Moreover, $\alpha_{3}$ is always the best alternative, while the worst is $\alpha_{5}$.

Table 5. The final results with $\theta=2.25$ and $1 \leq \lambda \leq 10$

\begin{tabular}{|c|c|c|c|}
\hline Parameter & Ranking of alternatives & The optimal one(s) & The worst one(s) \\
\hline$\lambda=1$ & $\alpha_{5} \prec \alpha_{2} \prec \alpha_{4} \prec \alpha_{1} \prec \alpha_{3}$ & $\alpha_{3}$ & $\alpha_{5}$ \\
\hline$\lambda=2$ & $\alpha_{5} \prec \alpha_{2} \prec \alpha_{4} \prec \alpha_{1} \prec \alpha_{3}$ & $\alpha_{3}$ & $\alpha_{5}$ \\
\hline$\lambda=4$ & $\alpha_{5} \prec \alpha_{2} \prec \alpha_{4} \prec \alpha_{1} \prec \alpha_{3}$ & $\alpha_{3}$ & $\alpha_{5}$ \\
\hline$\lambda=6$ & $\alpha_{5} \prec \alpha_{2} \prec \alpha_{4} \prec \alpha_{1} \prec \alpha_{3}$ & $\alpha_{3}$ & $\alpha_{5}$ \\
\hline$\lambda=8$ & $\alpha_{5} \prec \alpha_{2} \prec \alpha_{4} \prec \alpha_{1} \prec \alpha_{3}$ & $\alpha_{3}$ & $\alpha_{5}$ \\
\hline$\lambda=10$ & $\alpha_{5} \prec \alpha_{2} \prec \alpha_{4} \prec \alpha_{1} \prec \alpha_{3}$ & $\alpha_{3}$ & $\alpha_{5}$ \\
\hline
\end{tabular}

If $0<\theta \leq 5$ and $1 \leq \lambda \leq 10$, then different results can be produced as $\theta$ and $\lambda$ change (see Figures 3-8). It can be seen that these two parameters have insignificant influence on the rankings of alternatives. The best alternative is always $\alpha_{3}$ as $\theta$ and $\lambda$ change. However, if the two parameters are greater, then $\alpha_{1}$ will move closer to $\alpha_{3}$, and $\alpha_{2}$ will move closer to $\alpha_{5}$. Generally, DMs can choose different parameters according to the preferences. 


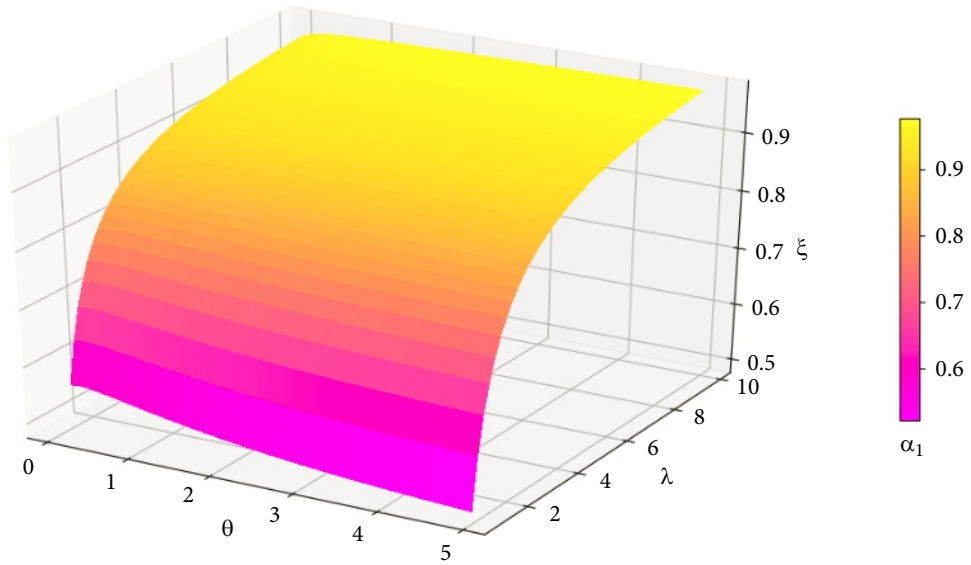

Figure 3. The final change trend of alternative $\alpha_{1}$ with $0<\theta \leq 5$ and $1 \leq \lambda \leq 10$

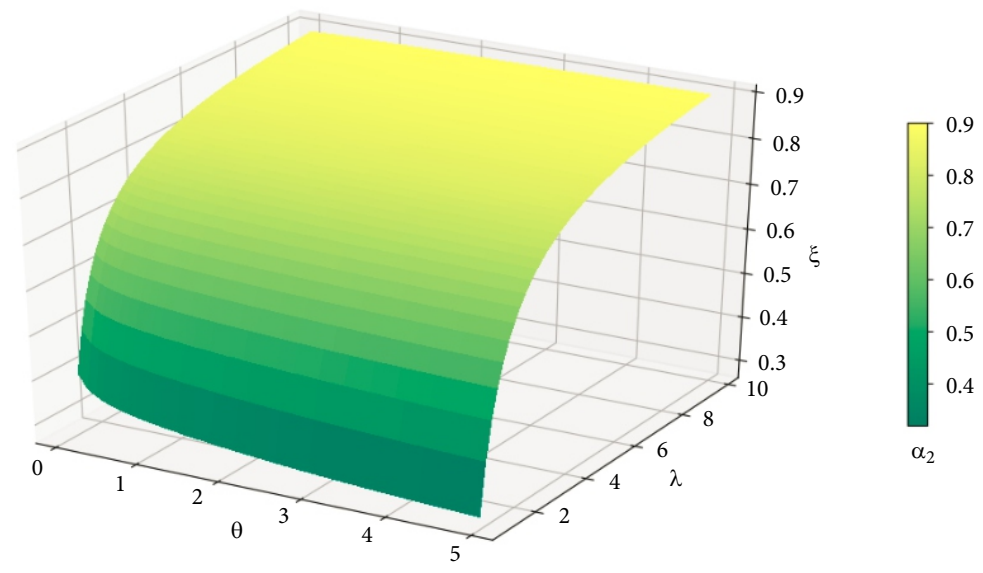

Figure 4. The final change trend of alternative $\alpha_{2}$ with $0<\theta \leq 5$ and $1 \leq \lambda \leq 10$

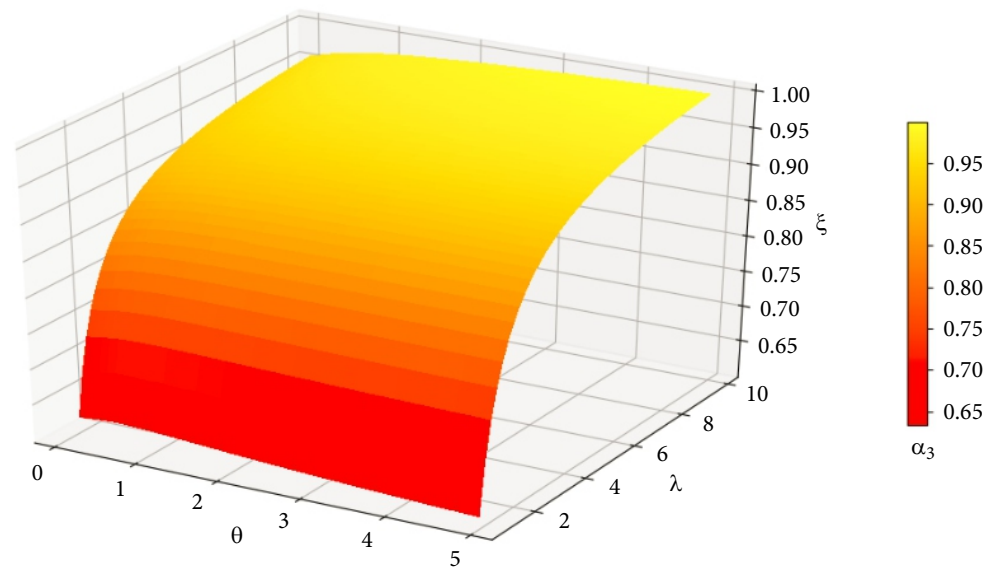

Figure 5. The final change trend of alternative $\alpha_{3}$ with $0<\theta \leq 5$ and $1 \leq \lambda \leq 10$ 

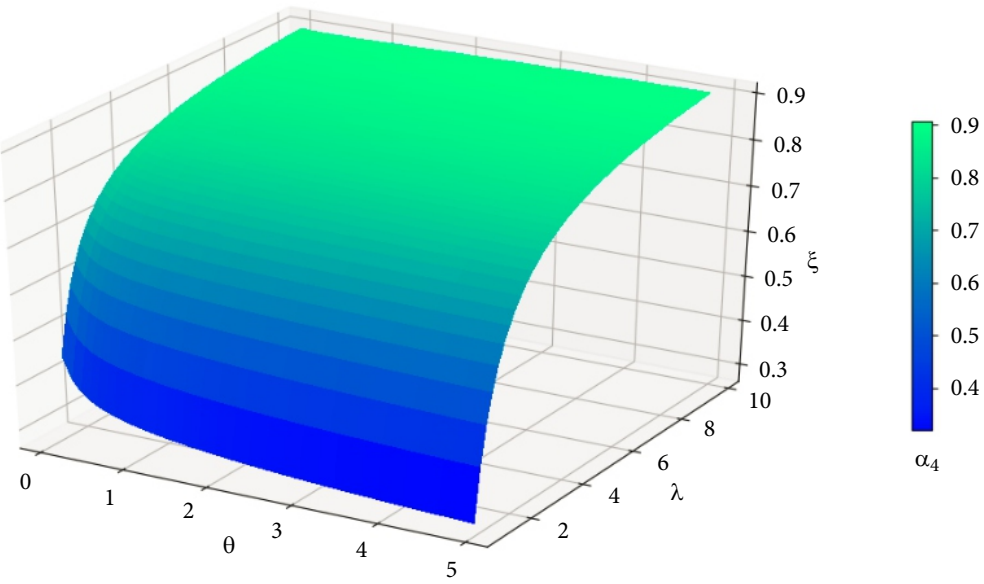

Figure 6. The final change trend of alternative $\alpha_{4}$ with $0<\theta \leq 5$ and $1 \leq \lambda \leq 10$
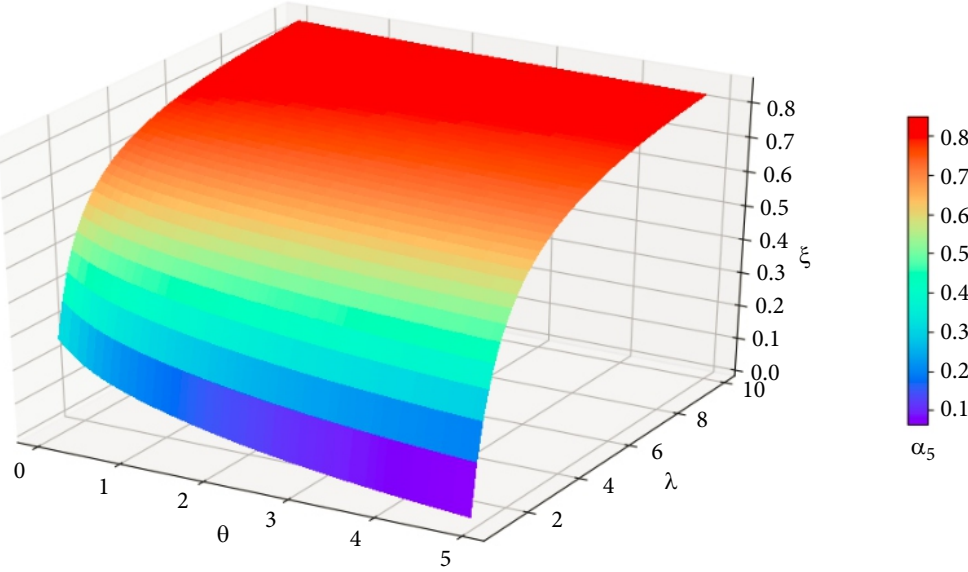

Figure 7. The final change trend of alternative $\alpha_{5}$ with $0<\theta \leq 5$ and $1 \leq \lambda \leq 10$
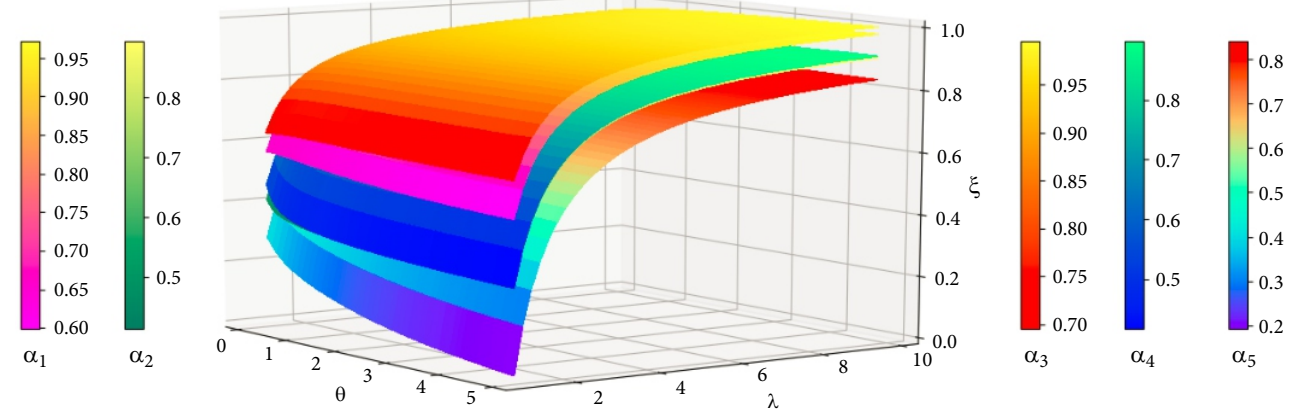

Figure 8. The final change trend of five alternatives with $0<\theta \leq 5$ and $1 \leq \lambda \leq 10$ 


\subsection{A comparative analysis}

To verify the effectiveness, results achieved by ANP-TODIM are compared with some existing methods based on measures and aggregation operators respectively (V. D. Nguyen \& X. T. Nguyen, 2018; Wei, 2016; Wang et al., 2018; Wang et al., 2019). As the compared methods cannot resolve situations where the weight information is fully unknown, the weights are provided as $w=(0.313,0.195,0.217,0.275)^{T}$ for the comparative analysis.

According to the method presented by V. D. Nguyen and X. T. Nguyen (2018), the distance measure between alternatives and ideal alternative can be calculated. The smaller the distance is, the better the alternative is. From the picture decision matrix presented in Table 4, the corresponding ideal solution of four criteria can be determined as follows: $\alpha_{1}^{*}=(0.65,0.06,0.14), \alpha_{2}^{*}=(0.62,0.07,0.17), \alpha_{3}^{*}=(0.64,0.09,0.15)$ and $\alpha_{4}^{*}=(0.67,0.06,0.05)$. Based on the picture fuzzy distance measures presented in Definition 3, we have

$$
\begin{aligned}
& D_{H}\left(\alpha_{1}, \alpha^{*}\right)=\sum_{j=1}^{4} w_{j} D_{H}\left(\alpha_{1 j}, \alpha_{j}^{*}\right)=\frac{1}{3}(|0.65-0.65|+|0.13-0.06|+|0.21-0.14|) \times 0.313+ \\
& \frac{1}{3}(|0.53-0.62|+|0.13-0.07|+|0.25-0.17|) \times 0.195+\frac{1}{3}(|0.64-0.64|+|0.11-0.09|+ \\
& |0.15-0.15|) \times 0.217+\frac{1}{3}(|0.66-0.67|+|0.09-0.06|+|0.18-0.05|) \times 0.275=0.0401 .
\end{aligned}
$$

Similarly,

$D_{H}\left(\alpha_{2}, \alpha^{*}\right)=0.0479 ; D_{H}\left(\alpha_{3}, \alpha^{*}\right)=0.0416 ; D_{H}\left(\alpha_{4}, \alpha^{*}\right)=0.0600 ; D_{H}\left(\alpha_{5}, \alpha^{*}\right)=0.0570$.

Since $D_{H}\left(\alpha_{4}, \alpha^{*}\right)>D_{H}\left(\alpha_{5}, \alpha^{*}\right)>D_{H}\left(\alpha_{2}, \alpha^{*}\right)>D_{H}\left(\alpha_{3}, \alpha^{*}\right)>D_{H}\left(\alpha_{1}, \alpha^{*}\right)$, thus the final ranking is $\alpha_{4} \prec \alpha_{5} \prec \alpha_{2} \prec \alpha_{3} \prec \alpha_{1}$.

If the other distances, i.e., $D_{E}, D_{H}^{m}$ and $D_{E}^{m}$, are used respectively, then the final rankings are $\alpha_{4} \prec \alpha_{5} \prec \alpha_{2} \prec \alpha_{3} \prec \alpha_{1}, \alpha_{5} \prec \alpha_{4} \prec \alpha_{1} \prec \alpha_{3} \prec \alpha_{2}$, and $\alpha_{5} \prec \alpha_{4} \prec \alpha_{1} \prec \alpha_{3} \prec \alpha_{2}$, respectively.

For the method presented by Wei (2016), the cross-entropy between the alternatives and the positive solution can be obtained and utilized to achieve the final ranking; however, for the method proposed by Wang et al. (2018), the corresponding weight can be obtained with $s=t=1$. Then, the total of the gains and losses of each alternative with all criteria with $\alpha=$ $\beta=0.88$ and $\gamma=2.25$ can be obtained. For the methods developed by Wang et al. (2019), the corresponding geometric aggregation operators is utilized to get the comprehensive preference, and the optimal alternative can be obtained by using score function. Hence, the five methods and the proposed approach are used together to analyze the same information, in which the results are listed in Table 6.

As can be seen, the final rankings are similar by using the compared methods (Wei, 2016; Wang et al., 2018) and the developed method. Although the positions of alternatives $\alpha_{2}$ and $\alpha_{4}$ can be changed by utilizing the method presented by Wei (2016), the optimal alternative is always $\alpha_{3}$. However, the results achieved by using distance measures presented by V. D. Nguyen and X. T. Nguyen (2018) are different from other methods, and the optimal one is $\alpha_{1}$ or $\alpha_{2}$, because the distance measures defined by V. D. Nguyen and X. T. Nguyen (2018) are unreasonable in some particular conditions as discussed earlier. Furthermore, the reason why the rankings calculated by using Wang et al. method (2019) are not consistent 
with those of the proposed method is that there exists a shortcoming in the operation rules of PFSs, i.e., the multiplication does not satisfy the closure. Hence, the corresponding geometric aggregation operator is also unreasonable, which may lead to unconvincing results.

Table 6. Results of a comparative analysis

\begin{tabular}{|c|c|}
\hline Methods & Ranking of alternatives \\
\hline Wei (2016) & $\alpha_{5} \prec \alpha_{4} \prec \alpha_{2} \prec \alpha_{1} \prec \alpha_{3}$ \\
\hline Wang et al. (2018) & $\alpha_{5} \prec \alpha_{2} \prec \alpha_{4} \prec \alpha_{1} \prec \alpha_{3}$ \\
\hline \multirow{2}{*}{ V. D. Nguyen and X. T. Nguyen (2018) } & $\begin{array}{c}\alpha_{4} \prec \alpha_{5} \prec \alpha_{2} \prec \alpha_{3} \prec \alpha_{1} \\
\left(D_{H} \text { and } D_{E}\right)\end{array}$ \\
\hline & $\begin{array}{c}\alpha_{5} \prec \alpha_{4} \prec \alpha_{1} \prec \alpha_{3} \prec \alpha_{2} \\
\left(D_{H}^{m} \text { and } D_{E}^{m}\right)\end{array}$ \\
\hline Wang et al. (2019) & $\alpha_{4} \prec \alpha_{5} \prec \alpha_{2} \prec \alpha_{3} \prec \alpha_{1}$ \\
\hline The proposed method & $\alpha_{5} \prec \alpha_{2} \prec \alpha_{4} \prec \alpha_{1} \prec \alpha_{3}$ \\
\hline
\end{tabular}

From the analyses presented above, some conclusions can be summarized. Firstly, the cross evaluation between the positive, neutral, and negative degrees is considered in the proposed novel normalized picture fuzzy distance measure. Secondly, the methods presented by Wei (2016), Wang et al. (2018), V. D. Nguyen and X. T. Nguyen (2018), and Wang et al. (2019) were all developed based on full rationality of a DM. However, the proposed approach based on ANP-TODIM considers a DM's psychological behavior, making it to be more widely applicable to actual decision-making problems. Thirdly, the developed approach can offer a flexible manner to effectively deal with some special MCDM problems, while the weight information is still fully unknown. Moreover, interrelationships among the criteria are considered by using the improved ANP to obtain the most important weights of criteria in tourism attraction recommendation. Therefore, the final results yielded by the proposed approach are more feasible and credible.

\section{Conclusions}

PFSs play a pivotal role in resolving MCDM problems, as they can precisely express a preference when there is uncertain or incomplete information. Hence, this paper investigated an integrated ANP-TODIM approach for tourism attraction recommendation with completely unknown weight information under picture fuzzy environment. The presented approach simultaneously considers a DMs' bounded rationality and interdependence among criteria. The weight of criteria is obtained by using the ANP method, and the TODIM method is applied to rank alternatives. Moreover, we testified the applicability and effectiveness of the developed approach using an illustrative example of an optimal tourism attraction recommendation and a comparative analysis. The sensitivity analysis was performed to discuss the influences of corresponding parameters on final results. 
The major contribution of this study can be concluded in the following. Firstly, the score and accuracy functions, as well as the corresponding comparison method of PFSs were defined. Secondly, the novel normalized picture fuzzy distance measure, which can consider cross-evaluation among positive degree, neutral degree, and negative degree, was proposed and some properties of distance measure were discussed. However, the existing picture fuzzy distance measure cannot take the cross-evaluation into account. Finally, based on the improved ANP method and proposed distance measure, this paper established an integrated ANP-TODIM approach to handle MCDM problems with fully unknown weight information. Thus, two mainly advantages of the proposed method can be summarized. One of the advantages of the proposed method is to use improved ANP method based on expert mean assessment to obtain the weight of criteria. The improved ANP method determines the confidence interval of experts in $[-2.3,2.3]$, which can avoid abnormal value in the evaluation process and ensure the objectivity and accuracy of the initial evaluation values. However, the majority of existing ANP methods does not consider experts' confidence interval. The other advantage is to develop the extended picture fuzzy TODIM method considering a DMs' psychological behavior. PFSs can express a DM' opinions accurately, including yes, abstain, no and refusal, which can make the data to be more adaptable with actual decision-making environment than FSs and their extensions. The TODIM method takes into account a DMs' attitude towards risk aversion. It can reflect a DMs' preference by adjusting for parameters in the decision-making process, and describe a DMs' psychological behavior, which is more consistent with the actual decision-making situation. Moreover, the method in this paper is proved to be more effective compared with some existing methods. In the future research, it is essential to study the other measures and applications of PFSs.

\section{Acknowledgements}

The authors are grateful for the comments provided by reviewers to improve this paper. This work is supported by the National Natural Science Foundation of China (Nos. 71701065, 71571193, 61603331 and 71801090), and the Postdoctoral Science Foundation of China (No. 2017M610511).

\section{Disclosure statement}

The authors declare that there is no conflict of interest regarding the publication of this paper.

\section{References}

Atanassov, K. (1986). Intuitionistic fuzzy sets. Fuzzy Sets and Systems, 20, 87-96. https://doi.org/10.1016/S0165-0114(86)80034-3

Barton, R. R., Nelson, B. L., \& Xie, W. (2014). Quantifying input uncertainty via simulation confidence intervals. Informs Journal on Compuing, 26(1), 74-87 (in Chinese).

https://doi.org/10.1287/ijoc.2013.0548 
Chang, K. L., Liao, S. K., Tseng, T. W., \& Liao, C. Y. (2015). An ANP based TOPSIS approach for Taiwanese service apartment location selection. Asia Pacific Management Review, 20(2), 49-55. https://doi.org/10.1016/j.apmrv.2014.12.007

Chatterjee, K., \& Kar, S. (2018a). A multi-criteria decision making method for renewable energy selection using Z-number in uncertain environment. Technological and Economic Development of Economy, 24(2), 739-764. https://doi.org/10.3846/20294913.2016.1261375

Chatterjee, K., \& Kar, S. (2018b). Supplier selection in telecom supply chain management: a fuzzy-rasch based COPRAS-G method. Technological and Economic Development of Economy, 24(2), 765-791. https://doi.org/10.3846/20294913.2017.1295289

Chen, J. Q., \& Tang, T. (2014). Research on simulation credibility of train operation control system based on improved FAHP method. Journal of the China Railway Society, 36(3), 59-66 (in Chinese).

Cuong, B. C., \& Kreinovich, V. (2013). Picture fuzzy sets-a new concept for computational intelligence problems. In Proceedings of 3rd World Congress on Information and Communication Technologies (WICT) (pp. 1-6), Hanoi, Vietnam. https://doi.org/10.1109/WICT.2013.7113099

Debnath, A., Bandyapadhyay, A., Roy, J., \& Kar, S. (2018). Game theory based multi criteria decisionmaking problem under uncertainty: a case study on Indian tea industry. Journal of Business Economics and Management, 19(1), 154-175. https://doi.org/10.3846/16111699.2017.1401553

Debnath, A., Roy, J., \& Kar, S. (2018). Measuring corporate social responsibility based on fuzzy analytic networking process-based balance scorecard model. International Journal of Information Technology \& Decision Making, 17(4), 1203-1235. https://doi.org/10.1142/S0219622018500232

Gomes, L. F. A. M., \& Lima, M. M. P. P. (1992a). TODIM: Basics and application to multicriteria ranking of projects with environmental impacts. Foundations of Computing and Decision science, 16(4), 113-127.

Gomes, L. F. A. M., \& Lima, M. M. P. P. (1992b). From modeling individual preferences to multicriteria ranking of discrete alternatives: a look at prospect theory and the additive difference model. Foundations of Computing and Decision Sciences, 17, 171-184.

Hu, J. H., Zhang, X. H., Yang, Y., Liu, Y. M., \& Chen, X. H. (2018). New doctors ranking system based on VIKOR method. International Transactions in Operational Research, 27(2), 1236-1261. https://doi.org/10.1111/itor.12569

Ji, P., Zhang, H. Y., \& Wang, J. Q. (2018). A projection-based TODIM method under multi-valued neutrosophic environments and its application in personnel selection. Neural Computing \& Applications, 29(1), 221-234. https://doi.org/10.1007/s00521-016-2436-Z

Joshi, R., Kumar, S., Gupta, D., \& Kaur, H. (2017). A Jensen- $\alpha$-norm dissimilarity measure for intuitionistic fuzzy sets and its applications in multiple attribute decision making. International Journal of Fuzzy Systems, 5, 1-15.

Kahneman, D., \& Tversky, A. (1979). Prospect theory: an analysis of decision under risk. Econometrica, 47(2), 263-291. https://doi.org/10.2307/1914185

Li, D. F., \& Cheng, C. T. (2002). New similarity measures of intuitionistic fuzzy sets and application to pattern recognition. Pattern Recognition Letters, 33, 221-225. https://doi.org/10.1016/S0167-8655(01)00110-6

Liu, H. W., \& Wang, G. J. (2007). Multi-criteria methods based on intuitionistic fuzzy sets. European Journal Operational Research, 179(1), 220-233. https://doi.org/10.1016/j.ejor.2006.04.009

Mishra, A. R., Jain, D., \& Hooda, D. S. (2017). Exponential intuitionistic fuzzy information measure with assessment of service quality. International Journal of Fuzzy Systems, 19(3), 788-798. https://doi.org/10.1007/s40815-016-0278-6

Ngan, R. T., Son, L. H., Cuong, B. C., \& Ali, M. (2018). H-max distance measure of intuitionistic fuzzy sets in decision making. Applied Soft Computing, 69, 393-425.

https://doi.org/10.1016/j.asoc.2018.04.036 
Nguyen, V. D., \& Nguyen, X. T. (2018). Some measures of picture fuzzy sets and their application in multi-attribute decision making. International Journal of Mathematical Sciences and Computing, 4(3), 23-41. https://doi.org/10.5815/ijmsc.2018.03.03

Peng, J. J., Wang, J. Q., \& Wu, X. H. (2016). Novel multi-criteria decision-making approaches based on hesitant fuzzy sets and prospect theory. International Journal of Information Technology \& Decision Making, 15(03), 621-643. https://doi.org/10.1142/S0219622016500152

Pramanik, S., Dalapati, S., Alam, S., \& Roy, T. K. (2018). NC-TODIM-based MAGDM under a neutrosophic cubic set environment. Information, 8(4), 149. https://doi.org/10.3390/info8040149

Saaty, T. L. (1996). Decision making with dependence and feedback: The analytic network process. International, 95(2), 129-157.

Shen, K. W., Li, L., \& Wang, J. Q. (2019). Circular economy model for recycling waste resources under government participation: a case study in industrial waste water circulation in China. Technological and Economic Development of Economy.

Singh, P. (2015). Correlation coefficients for picture fuzzy sets. Journal of Intelligent \& Fuzzy Systems, 28(2), 591-604.

Son, L. H. (2017). Measuring analogousness in picture fuzzy sets: from picture distance measures to picture association measures. Fuzzy Optimization \& Decision Making, 16, 1-20.

https://doi.org/10.1007/s10700-016-9249-5

Thong, P. H. (2016a). Picture fuzzy clustering for complex data. Engineering Applications of Artificial Intelligence, 56, 121-130. https://doi.org/10.1016/j.engappai.2016.08.009

Thong, P. H. (2016b). A novel automatic picture fuzzy clustering method based on particle swarm optimization and picture composite cardinality. Knowledge-Based Systems, 109, 48-60.

https://doi.org/10.1016/j.knosys.2016.06.023

Tian, C., Peng, J. J., Zhang, S., Zhang, W. Y., \& Wang, J. Q. (2019). Weighted picture fuzzy aggregation operators and their applications to multi-criteria decision-making problems. Computers \& Industrial Engineering, 137, 106037. https://doi.org/10.1016/j.cie.2019.106037

Wang, J. Q., Han, Z. Q., \& Zhang, H. Y. (2014). Multi-criteria group decision-making method based on intuitionistic interval fuzzy information. Group Decision and Negotiation, 23, 715-733.

https://doi.org/10.1007/s10726-012-9316-4

Wang, L., Peng, J. J., \& Wang, J. Q. (2018). A multi-criteria decision-making framework for risk ranking of energy performance contracting project under picture fuzzy environment. Journal of Cleaner Production, 191, 105-118. https://doi.org/10.1016/j.jclepro.2018.04.169

Wang, L., Wang, X. K., Peng, J. J., \& Wang, J. Q. (2020). The differences in hotel selection among various types of travellers: A comparative analysis with a useful bounded rationality behavioural decision support model. Tourism Management, 76, 103961. https://doi.org/10.1016/j.tourman.2019.103961

Wang, L., Zhang, H. Y., Wang, J. Q., Wu, G. F. (2019). Picture fuzzy multi-criteria group decisionmaking method to hotel building energy efficiency retrofit project selection. RAIRO-Operations Research. https://doi.org/10.1051/ro/2019004

Wang, R., Wang, J., Gao, H., \& Wei, G. W. (2019). Methods for MADM with picture fuzzy Muirhead mean operators and their application for evaluating the financial investment risk. Symmetry, 11(1), 6 . https://doi.org/10.3390/sym11010006

Wang, W. Z., \& Liu, X. W. (2012). Intuitionistic fuzzy information aggregation using Einstein operations. IEEE Transactions on Fuzzy Systems, 20, 923-938. https://doi.org/10.1109/TFUZZ.2012.2189405

Wang, W., \& Xin, X. (2005). Distance measure between intuitionistic fuzzy sets. Pattern Recognition Letters 26(13), 2063-2069. https://doi.org/10.1016/j.patrec.2005.03.018

Wei, G. W. (2016). Picture fuzzy cross-entropy for multiple attribute decision making problems. Journal of Business Economics and Management, 17(4), 491-502.

https://doi.org/10.3846/16111699.2016.1197147 
Wen, Y. M., Shi, Y. F., Cai, G. Y., Miao, Y. Q., \& Long, G. (2014). A survey of personalized travel recommendation. Computer Science, Z11.

Wu, Y. N., Zhang, B. Y., Xu, C. B., \& Li, L. W. Y. (2018). Site selection decision framework using fuzzy ANP-VIKOR for large commercial rooftop PV system based on sustainability perspective. Sustainable Cities and Society, 40, 454-470. https://doi.org/10.1016/j.scs.2018.04.024

$\mathrm{Xu}, \mathrm{Z}$. S. (2007). Intuitionistic fuzzy aggregation operations. IEEE Transactions on Fuzzy Systems, 15, 1179-1187. https://doi.org/10.1109/TFUZZ.2006.890678

Yang, L. Z., Gong, X. Y., Wang, X. J., \& An, S. Q. (2014). Generalized exponential entropy on intuitionistic fuzzy sets. Applied Mechanics \& Materials, 556-562, 4097-4102. https://doi.org/10.4028/www.scientific.net/AMM.556-562.4097

Yang, Y., Hu, J. H., Liu, Y. M., \& Chen, X. H. (2018). Doctor recommendation based on an intuitionistic normal cloud model considering patient preferences. Cognitive Computation, 1-19. https://doi.org/10.1007/s12559-018-9616-3

Yu, S. M., Wang, J., \& Wang, J. Q. (2018). An extended TODIM approach with intuitionistic linguistic numbers. International Transactions in Operational Research, 25(3), 781-805. https://doi.org/10.1111/itor.12363

Zadeh, L. A. (1965). Fuzzy sets. Information and Control, 8, 338-356. https://doi.org/10.1016/S0019-9958(65)90241-X

Zhang, G., Wang, J. Q., \& Wang, T. L. (2019). Multi-criteria group decision-making method based on TODIM with probabilistic interval-valued hesitant fuzzy information. Expert Systems, 36(4), 12424. https://doi.org/10.1111/exsy.12424

Zhang, X. L., \& Xu, Z. S. (2014). The TODIM analysis approach based on novel measured functions under hesitant fuzzy environment. Knowledge-Based Systems, 61, 48-58. https://doi.org/10.1016/j.knosys.2014.02.006

Zhang, X. Y., Wang, J. Q., \& Hu, J. H. (2018). On novel operational laws and aggregation operators of picture 2-tuple linguistic information for MCDM problems. International Journal of Fuzzy Systems, 20(3), 958-969. https://doi.org/10.1007/s40815-017-0441-8

Zhang, Y. P., Wang, Y. H., \& Yang, N. (2018). On the risk assessment method of balise system based on the ANP and fuzzy evidence theory. Journal of Safety and Environment, 18(2), 434-440 (in Chinese). 